Subscriber access provided by University of East Anglia Library

\title{
Article
}

\section{Excess imidacloprid exposure causes the heart tube malformation of chick embryos}

Lin-rui Gao, Shuai Li, Jing Zhang, Chang Liang, En-ni Chen, Shi-yao

Zhang, Manli Chuai, Yongping Bao, Guang Wang, and xuesong Yang

J. Agric. Food Chem., Just Accepted Manuscript • DOI: 10.1021/acs.jafc.6b03381 • Publication Date (Web): 28 Oct 2016

Downloaded from http://pubs.acs.org on November 2, 2016

\section{Just Accepted}

"Just Accepted" manuscripts have been peer-reviewed and accepted for publication. They are posted online prior to technical editing, formatting for publication and author proofing. The American Chemical Society provides "Just Accepted" as a free service to the research community to expedite the dissemination of scientific material as soon as possible after acceptance. "Just Accepted" manuscripts appear in full in PDF format accompanied by an HTML abstract. "Just Accepted" manuscripts have been fully peer reviewed, but should not be considered the official version of record. They are accessible to all readers and citable by the Digital Object Identifier (DOI®). "Just Accepted" is an optional service offered to authors. Therefore, the "Just Accepted" Web site may not include all articles that will be published in the journal. After a manuscript is technically edited and formatted, it will be removed from the "Just Accepted" Web site and published as an ASAP article. Note that technical editing may introduce minor changes to the manuscript text and/or graphics which could affect content, and all legal disclaimers and ethical guidelines that apply to the journal pertain. ACS cannot be held responsible for errors or consequences arising from the use of information contained in these "Just Accepted" manuscripts. 

Excess imidacloprid exposure causes the heart tube malformation of chick embryos

3

4 Lin-rui Gao ${ }^{1 \#}$, Shuai Li ${ }^{1 \#}$, Jing Zhang ${ }^{1}$, Chang Liang ${ }^{1}$, En-ni Chen ${ }^{1}$, Shi-yao Zhang ${ }^{1}$, Manli Chuai ${ }^{2}$, Yong-ping Bao ${ }^{3}$, Guang Wang ${ }^{1 *}$, Xuesong Yang ${ }^{1 *}$

6

${ }^{1}$ Division of Histology and Embryology, Key Laboratory for Regenerative Medicine of the Ministry of Education, Medical College, Jinan University, Guangzhou 510632,

${ }^{2}$ Division of Cell and Developmental Biology, University of Dundee, Dundee, DD1 $5 E H, U K$

${ }^{3}$ Norwich Medical School, University of East Anglia, Norwich, Norfolk, NR4 7UQ,

$$
\text { " contribute to the work equally }
$$

*The corresponding authors :

Xuesong Yang:Tel: +86-20-85228316. E-mail address: yang_xuesong@126.com;or Guang Wang: Tel: +86-20-85220254. E-mail address: t_wangguang@jnu.edu.cn, wangguang7453@126.com 1 22 


\section{Abstract}

As a neonicotinoid pesticide, imidacloprid is widely used to control sucking insects on agricultural planting and fleas on domestic animals. However, the extent to which imidacloprid exposure has an influence on cardiogensis in early embryogenesis is still poorly understood. In vertebrates, the heart is the first organ to be formed. In this study to address whether or not imidacloprid exposure affects early heart development, the early chick embryo has been used as an experimental model because of the accessibility of chick embryo at its early developmental stage. The results demonstrate that exposure of the early chick embryo to imidacloprid caused malformation of heart tube. Furthermore, the data reveal that down-regulation of GATA4, Nkx2.5 and BMP4 and up-regulation of Wnt3a led to aberrant cardiomyocyte differentiation. In addition, imidacloprid exposure interfered with basement membrane (BM) breakdown, E-cadherin/Laminin expression and mesoderm formation during the epithelial-mesenchymal transition (EMT) in gastrula chick embryos. Finally, the DiI-labeled cell migration trajectory indicated that imidacloprid restricted the cell migration of cardiac progenitors to primary heart field in gastrula chick embryos. A similar observation was also obtained from the cell migration assay of scratch wounds in vitro. Additionally, imidacloprid exposure negatively affected the cytoskeleton structure and expression of corresponding adhesion molecules. Taken together, these results reveal that the improper EMT, cardiac progenitor migration and differentiation are responsible for imidacloprid exposure-induced malformation of heart tube during chick embryo development. 
46 Keywords: Imidacloprid; chick embryo; heart tube; EMT; cardiac progenitor 47 migration; differentiation.

48 


\section{Introduction}

Organogenesis requires the precise layout of multiple cell types into a specific three-dimensional architecture that is essential for normal organ formation. During embryonic organ development, an obligatory process is tissue fusion, such as that of the optic cup, palate, heart, neural tube, eyelids and body wall ${ }^{1,2}$. Tissue fusion appears to occur in numerous organs. Our previous study demonstrated that the deficiency of specific transcription factors and signaling molecules could exhibit the fusion defects in many organs, for instance, in neural tube defects ${ }^{3}$ and cardiac bifida

4. As a model of organogenesis, cardiogenesis involves a series of morphogenetic steps. In vertebrates, the heart develops from three distinct pools of cardiac progenitors: the cardiac precursor in splanchnic mesoderm (primary and secondary heart field), cardiac neural crest and the pro-epicardium. From the perspective of morphological alteration, it is chronologically composed of primary heart tube fusion, cardiac looping and accretion, cardiac septation and coronary vasculogenesis ${ }^{5}$. The primary heart field gives rise to the major structures of the heart, including the atrias and ventricles, while the secondary heart field contributes to the cardiac outflow tracts 6. Myocardial progenitors undergo Epithelial-Mesenchymal Transition (EMT), proliferate, differentiation and migration into the primary heart field in the process of heart tube formation. EMT is a morphogenetic transition process in which cells lose their epithelial characteristics and gain mesenchymal properties underlying the alterations of adheren junction (AJs), tight junction (TJs) and gap junction (GJs) ${ }^{7,8}$.

In the formation of primary heart fields, the precardiac cells initially migrate out of 
the anterior primitive streak at the gastrula stage and then move symmetrically into crescent location $^{9-11}$. Cell migration, proliferation and differentiation are guided by its micro-environment ${ }^{12}$.

The morphogenesis of chick cardiac looping involves four phases: pre-looping phase (HH8-9); C-shaped bend (HH9 $\left.{ }^{+}-13\right)$; S-shaped heart loop (HH14-16) and primitive outflow tract formation (about 4.5 days). Within days 6-14, expansion and growth of the ventricular wall benefit principally from cardiomyocyte proliferation in the compact myocardium. At day 14.0, cardiac neural crest cells (CNCs) give rise to the adventitia of the large veins and the coronary arteries. In this context, any disruption to cardiac precursor cell migration and differentiation during cardiogenesis may result in congenital heart malformations.

Heart development is a complex process that is tightly regulated through spatio-temporal gene expression and cell-cell interaction. In previous studies of heart tube assembly in the chick embryo, we have reported that fibroblast growth factor (FGF) signaling, through an endoderm-derived signal, is required for regulating pro-cardiac mesoderm cell migration ${ }^{10,13}$. Additionally, bone morphogenetic protein 2 (BMP2) is released from the anterior endoderm and Wnt antagonists are essential for precardiac mesoderm cells to differentiate into mature cardiomyocytes during cardiomyogenesis ${ }^{14-16}$. Furthermore, transcription factors Nkx-2.5, GATA4, myocardin and TBX5 have crucial roles in dictating morphogenesis and differentiation of the heart ${ }^{16,17}$. Vascular endothelial growth factor (VEGF) also plays a vital role in the angiogenic expansion of the early network ${ }^{18}$. 
The

neonicotinoid

pesticide,

imidacloprid, 1-((6-Chloro-3-pyridinyl)methyl)-N-nitroimidazolidinimine, has been extensively used to control sucking insects, termites, soil insects on crops ${ }^{19}$ and fleas on domestic animals 20,21 . Various products containing this chemical, including liquids, granules, dusts and packages, have been sold in the US since 1994. In the EU, use of imidacloprid was restricted for 2 years in 2013 because research showed a link between imidacloprid and bee death (EASAC 2015, Ecosystem services, agriculture and neonicotinoids). As a systemic insecticide, imidacloprid products are usually sprayed on soil and leaves, and then spread to the plant's stems, leaves, fruit and flowers 22,23 . Imidacloprid can then penetrate into the nervous system of sucking insects and combine selectively with nicotinic acetylcholine receptors (nAChR), producing toxic effects ${ }^{24}$. When insects consume plants treated with imidacloprid products, their nervous systems are damaged leading to death. Due to steric conditions at the nAChR, imidacloprid has much lower toxicity to mammals. However, humans can be exposed to imidacloprid products via skin/eye contact or through consumption or inhalation when handling the pesticide or an animal recently exposed to imidacloprid. The toxicity of imidacloprid in human adults is due to disruption of nervous system signal transduction ${ }^{25}$. Once humans are exposed, imidacloprid products can cross the lining of the intestine and be transported to the whole body through circulation of the blood. However, little is known about its potential toxic effects on early embryo development apart from a few reports on human health such as reproductive ability. Currently, increasing attention is being paid to the toxic effects 
115 of pesticides on embryo development, including cardiovascular system. Unfortunately, 116 as yet there is no direct evidence of toxicological effects on cardiogenesis or

117 corresponding mechanisms. In this study, a chick embryo model ${ }^{26}$ has been used to 118 investigate whether or not imidacloprid could affect cardiogenesis and, if so, to 119 elucidate the underlying cellular and molecular mechanism.

\section{Materials and methods}

\section{Chick manipulations}

Fertilized leghorn eggs were acquired from the Avian Farm of South China Agriculture University (Guangzhou, China). Two approaches were employed to carry out the imidacloprid exposure in this study. The imidacloprid powder was dissolved in dimethyl sulfoxide (DMSO), 0.1\% DMSO was used as control to observe the potential effect of the solvent.

For imidacloprid exposure at the early embryonic stage, Hamburger-Hamilton (HH) stage 0 chick embryos from fertilized eggs were incubated with either $0.1 \%$ DMSO (control) or $500 \mu \mathrm{M}$ imidacloprid ${ }^{27}$ in early chick (EC) culture medium in a humidified incubator (Yiheng Instruments, Shanghai, China) at $38^{\circ} \mathrm{C}$ and $70 \%$ humidity until the chick embryos developed to the HH10 stage. Alternatively, $500 \mu \mathrm{M}$ imidacloprid was directly applied to one side of the gastrula-stage embryos, with the other side being exposed to $0.1 \%$ DMSO as a control.

For imidacloprid exposure at a later embryonic stage, HH4 chick embryos were exposed to either $0.1 \%$ DMSO (control) or $500 \mu \mathrm{M}$ imidacloprid through injection 
137 into windowed eggs in vivo and then further incubated for 4.5 days and 14 days. The

138 experiments were performed in triplicate with 20 eggs assigned to each group, and

139 surviving embryos were harvested for further assessment.

\section{In situ hybridization}

141 Whole-mount in situ hybridization of chick embryos was performed according to 142 a standard in situ hybridization protocol ${ }^{28}$. Briefly, digoxigenin-labeled probes were 143 synthesized for VMHC ${ }^{29}$, GATA5 ${ }^{30}$, BMP2 and NKX2.5 (supplied by Dr. Thomas M. 144 Schultheiss). The whole-mount stained embryos were photographed and then frozen 145 sections prepared on a cryostat microtome (LeicaCM1900) at a thickness of 15-20 $146 \mathrm{~mm}$.

\section{Immunofluorescent staining}

148 Chick embryos were harvested at the end of the experiment and fixed overnight 149 in $4 \%$ paraformaldehyde at $4^{\circ} \mathrm{C}$. Whole-mount embryos were immunofluorescently 150 stained using MF20 (1:500, DSHB, USA), E-cadherin (1:50, BD Transduction 151 Laboratories, USA), Laminin (1:100, DSHB, USA) antibodies. Briefly, the fixed 152 embryos were incubated with these primary antibodies at $4^{\circ} \mathrm{C}$ overnight on a rocker. 153 Following extensive washing, the embryos were incubated with the appropriate 154 anti-mouse IgG conjugated to Alexa Fluor 488 or anti-rabbit IgG conjugated to Alexa 155 Fluor 555 (1:1000, Invitrogen, USA), overnight at $4^{\circ} \mathrm{C}$ on a rocker. All embryos were

156 finally counterstained with DAPI (1:1000, Invitrogen, USA) at room temperature for 1571 hour. 

Total RNA was isolated from HH4, HH8 chick embryos using a Trizol kit

160 (Invitrogen, USA) according to the manufacturer's instructions. First-strand cDNA 161 was synthesized to a final volume of $25 \mu$ using SuperScript RIII first-strand 162 (Invitrogen, USA). Following reverse transcription, PCR amplification of the cDNA was performed as described previously. The primers used for RT-PCR are provided in the Figure S3. The PCR reactions were performed on a Bio-Rad S1000TM Thermal cycler (Bio-Rad, USA). The final reaction volume was $50 \mu \mathrm{l}$ composed of $1 \mu \mathrm{l}$ of first-strand cDNA, $25 \mu \mathrm{M}$ forward primer, $25 \mu \mathrm{M}$ reverse primer, $10 \mu \mathrm{l}$

PrimeSTARTM Buffer $\left(\mathrm{Mg}^{2+}\right.$ plus), $4 \mu \mathrm{l}$ dNTPs Mixture (TaKaRa, Japan), $0.5 \mu \mathrm{l}$

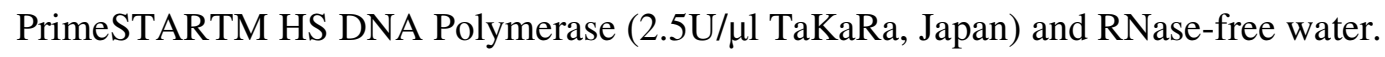

The cDNA was amplified for 30 cycles. One round of amplification was performed at $94^{\circ} \mathrm{C}$ for $30 \mathrm{~s}, 30 \mathrm{~s}$ at $58^{\circ} \mathrm{C}$, and $30 \mathrm{~s}$ at $72^{\circ} \mathrm{C}$. The PCR products $(20 \mu \mathrm{l})$ were resolved using $1 \%$ agarose gels (Biowest, Spain) in $1 \times$ TAE buffer $(0.04 \mathrm{M}$ Trisacetate and 0.001 MEDTA) and 10,000x GeneGreen Nucleic Acid Dye (Tiangen, China) solution.

173 The resolved products were visualized using a transilluminator (Syngene, UK) and 174 photographs captured using a computer-assisted gel documentation system (Syngene). The housekeeping gene GAPDH was run in parallel to confirm that equal amounts of RNA were used in each reaction. The ratio between intensity of the fluorescently

177 stained bands corresponding to genes and GAPDH was calculated to quantify the 178 level of the transcripts for those genes mRNAs. The RT-PCR result was representative 179 of three independent experiments.

\section{Cell trace with DiI}



perchlorate (DiI, Molecular Probes, Inc.) was used to label small groups of primitive streak cells. A $2.5 \%$ stock solution of DiI was diluted in ethanol, 1:10 in $0.3 \mathrm{M}$

184 sucrose, and injected into the anterior primitive streak of HH3 chick embryo by air pressure through a micropipette, which was pulled from a $1 \mathrm{~mm}$ glass capillary in a vertical micropipette puller (WD-2, Chengdu Instrument Company). In general, each labeled tissue in the anterior primitive streak contained approximately 10-30 cells.

\section{Cell lines and culture}

The H9c2 rat cardiac myoblast cell line was obtained from ATCC (American Type Culture Collection, CLR-1446, USA). The cells were cultured in a humidified incubator with $5 \% \mathrm{CO}_{2}$ at $37^{\circ} \mathrm{C}$ in six-well plates $\left(1 \times 10^{6}\right.$ cells $\left./ \mathrm{ml}\right)$ containing DMEM (Gibco, Gaithersburg, MD, USA), supplemented with 10\% fetal bovine serum (Gibco,

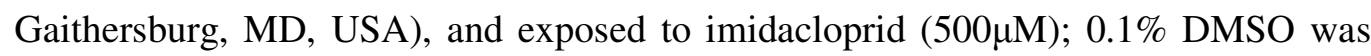
used as a control. The cells were photographed using an inverted fluorescence microscope (Nikon, Tokyo, Japan) with NIS-Elements F3.2 software. After 12 hours incubation, immunofluorescent staining against phalloidin (F-actin, 1:1000, Invitrogen, Waltham, MA, USA) and anti-Myh7 (1:100, Proteintech, USA) was performed on the incubated $\mathrm{H} 9 \mathrm{c} 2$ cells. A minimum of 5 images were assayed per treatment group. DAPI (1:1000, Invitrogen, USA) was used as counterstain.

\section{Migration assay}

H9c2 cells were seeded in 6-well plates with DMEM (10\% FBS) medium. At confluency, a wound was induced by scratching the monolayer with a $10-\mu l$ pipette tip. 
203 The cells were then washed 3 times with sterile PBS. H9c2 cells were incubated in

204 serum-free DMEM medium with $500 \mathrm{uM}$ or $0.1 \%$ DMSO under $5 \% \mathrm{CO}_{2}$ conditions.

205 Images were acquired at $12 \mathrm{~h}$ and $24 \mathrm{~h}$ post-scratching. At least 3 wells were analyzed

206 in each treatment group and the images were taken using an inverted microscope

207 (Nikon Eclipse Ti-U, Japan).

Western blot

Chick embryos (HH4 and HH7) were collected and lysed with CytoBuster ${ }^{\mathrm{TM}}$ Protein Extraction Reagent (\#71009, Novagen). The total protein concentration was established using a BCA quantification kit (BCA01, DingGuo BioTECH, CHN). Samples containing equal amounts of protein were resolved by SDS-PAGE and then transferred to PVDF membranes (Bio-Rad). The membranes were blocked with 5\% Difco $^{\mathrm{TM}}$ skim milk (BD) and then incubated with primary and secondary antibodies.

215 The antibodies used were TBX5, GATA4 and GATA6 (Abcam USA), 216 HRP-conjugated anti-mouse IgG and anti-rabbit IgG (Cell Signaling Technology, 217 USA). All primary and secondary antibodies used were diluted to 1:1000 and 1:2000

218 in 5\% skim milk, respectively. The protein bands of interest were visualized using an ECL kit (\#34079, Thermo Fischer Scientific Inc.) and GeneGnome5 (Syngene). The staining intensity of the bands was determined and analyzed using Quantity One 221 software (Bio-Rad).

\section{2}

\section{Photography}

Following immunofluorescent staining or in situ hybridization, the whole-mount embryos were photographed using a stereo-fluorescent microscope (Olympus MVX10) 
and associated Olympus software package Image-Pro Plus 7.0. The embryos were sectioned into $14 \mu \mathrm{m}$-thick slices using a cryostat microtome (Leica CM1900) and the sections were then photographed with an epi-fluorescent microscope (Olympus LX51, Leica DM 4000B) and CN4000 FISH Olympus software package.

\section{Data analysis}

The thickness of ventricular wall and trabecular muscle and the distance of wound closure in wound healing experiments as well as the lengths of the long and short axes were all quantified with Image-Pro Plus 6.0. The cell trace with DiI experiments, $\mathrm{Dil}^{+}$cells were manually counted with Image-Pro Plus 6.0. Statistical analyses for all the experimental data was performed using a SPSS 13.0 statistical package program for Window. The data were presented as mean \pm SD. Statistical significance were determined using paired T-test, independent samples T-test or one-way analysis of variance (ANOVA). ${ }^{*} \mathrm{p}<0.05, * * \mathrm{p}<0.01$ and $* * * \mathrm{p}<0.001$ indicate statistically significance between control and drug-treated groups. $\mathrm{P}<0.05$ was considered to be significant.

\section{Results}

Imidacloprid exposure increases cardiac malformation during chick cardiogenesis

The heart is the first functional organ in the developing embryo. There are three crucial phases in the development of heart formation: 2-, 4.5- and 14-day (Fig. 1A). To investigate the effects of excess imidacloprid exposure on heart tube formation in chick embryos, we cultured the embryos as shown in Figure S1. In the first place, we 
found that $35 \%(\mathrm{n}=28 / 80), 42.5 \%(\mathrm{n}=34 / 80)$ and $50 \%(\mathrm{n}=40 / 80)$ of $500 \mu \mathrm{M}$ imidacloprid-treated chick embryos had died after 2, 4.5 and 14 days incubation, respectively. Corresponding mortalities were only 5\% $(n=3 / 60), 6.67 \%(n=4 / 60)$ and $8.33 \%(\mathrm{n}=5 / 60)$ in the $0.1 \%$ DMSO-treated chick embryos (Fig. 1B). Our results showed that the growth of imidacloprid-treated embryos is slightly faster than $0.1 \%$ DMSO-treated ones at $21 \mathrm{~h}$ and, conversely, slightly delayed at $48 \mathrm{~h} .(21 \mathrm{~h}:$ DMSO $=$ $1345 \pm 74.43 \mu \mathrm{m}$, imidacloprid $=1629 \pm 82.45 \mu \mathrm{m}, \mathrm{P}<0.05 ; 48 \mathrm{~h}:$ DMSO $=4183 \pm$ $45.57 \mu \mathrm{m}$, imidacloprid $=3866 \pm 56.58 \mu \mathrm{m}, \mathrm{P}<0.001 ; \mathrm{n}=40$ for each groups; Figs 1C-C5, D).

The average number of somites in imidacloprid-treated group at $48 \mathrm{~h}$ was about 10 pairs compared to 12 pairs in $0.1 \%$ DMSO-treated group $(\mathrm{DMSO}=12.43 \pm 0.17$, imidacloprid $=10.03 \pm 0.15, \mathrm{n}=40$ for each groups, $\mathrm{P}<0.05$; Fig. 1E). Next, E4.5 imidacloprid-treated whole embryos weights were obviously lower than $0.1 \%$ DMSO-treated ones $(\mathrm{DMSO}=0.26 \pm 0.03 \mathrm{~g}, \mathrm{n}=10$, imidacloprid $=0.19 \pm 0.01 \mathrm{~g}, \mathrm{n}=$ 34, $\mathrm{P}<0.01$; Fig. $1 \mathrm{~F}) . \mathrm{H} \& \mathrm{E}$ staining revealed that the thicknesses of the ventricular walls $(\mathrm{DMSO}=47.52 \pm 0.95 \mu \mathrm{m}, \mathrm{n}=10$, imidacloprid $=28.85 \pm 0.72 \mu \mathrm{m}, \mathrm{n}=14, \mathrm{P}<$ 0.001) and the trabecular muscles were both reduced in imidacloprid-treated group compared with $0.1 \%$ DMSO-treated controls $(\mathrm{DMSO}=25.27 \pm 0.56 \mu \mathrm{m}, \mathrm{n}=10$, imidacloprid $=12.57 \pm 0.31 \mu \mathrm{m}, \mathrm{n}=14, \mathrm{P}<0.001$; Figs. 1G, G1-G2, H, H1-H2; I, J). Additionally, the size and weight of imidacloprid-treated hearts were smaller and lighter than those of 14-day 0.1\% DMSO-treated embryos (DMSO $=0.08 \pm 0.01 \mathrm{~g}, \mathrm{n}$ $=10$; imidacloprid $=0.07 \pm 0.01 \mathrm{~g}, \mathrm{n}=16, \mathrm{P}<0.05$; Figs. $1 \mathrm{~K}, \mathrm{~L}, \mathrm{M})$. The weight of 
whole embryo showed a similar tendency $(\mathrm{DMSO}=7.60 \pm 0.31 \mathrm{~g}, \mathrm{n}=10$, imidacloprid $=6.03 \pm 0.29 \mathrm{~g} . \mathrm{n}=16, \mathrm{P}<0.01 ;$ Fig. $1 \mathrm{~N}$ ). Transverse sections (Figs. $1 \mathrm{~K} 1, \mathrm{~L} 1)$ and histograms established that the right ventricular wall (RV) was dramatically thicker $(\mathrm{DMSO}=409.10 \pm 24.73 \mu \mathrm{m}, \mathrm{n}=10$, imidacloprid $=598.20 \pm$ $36.10 \mu \mathrm{m} . \mathrm{n}=16, \mathrm{P}<0.001$; Fig. 1O) whilst there was no significant difference in the left ventricular wall (LV) and interventricular septum (ISV).

Some atypical C-looping heart tube was evident when imidacloprid-treated embryos reached HH10. According to the phenotype features, we divided them into four classifications: normal (Figs. 2A, B), mild (Figs. 2A1, B1), intermediate (Figs. 2A2, B2) and severe (Figs. 2A3, B3), and all were stained with MF20 antibody and ventricular myosin heavy chain (VMHC) probe, respectively. In the $0.1 \%$ DMSO-treated embryonic heart, the heart tubes are fully C-looped (normal $=100 \%, \mathrm{n}$ $=80 / 80$ ), while abnormal morphological looping of heart tube occurred in the imidacloprid-treated groups $($ normal $=13.6 \%, \mathrm{n}=8 / 59$, mild $=39 \%, \mathrm{n}=23 / 59$, intermediate $=28.8 \%, \mathrm{n}=17 / 59$, severe $=18.6 \%, \mathrm{n}=11 / 59 ;$ Fig. $2 \mathrm{C})$. At stage HH10, the C-shape loop of the heart tube has formed in control embryos (Fig. 2D) as indicated by MF20 immunofluorescent-staining (Fig. 2E). The single cavity of the heart tube was also evident in corresponding transverse sections for these $0.1 \%$ DMSO-treated embryos (Figs. 2F, F1-F3). In contrast, some heart tubes of the HH10 imidacloprid-treated chick embryos presented in cardiac bifida (Fig. 2G), as shown in the MF20 immunofluorescently-stained heart tubes (Fig. 2H) and corresponding transverse section of the heart tubes. The two cavities were found in the transverse 
291 sections of the heart tubes (Figs. 2I, I1-I3).

292 Imidacloprid treatment represses cardiomyocyte differentiation

293 Figure 3A reveals the principal signaling pathways (Wnt, BMP\&FGF and VEGF)

294 involved in the regulation of cardiomyocyte differentiation at cardiac crescent stages

295 (HH7-8). To explore whether imidacloprid exposure affects these crucial gene

296 expressions of cardiomyocyte formation, we firstly exposed imidacloprid to one side

297 of the embryos, using the other side as control. This approach has been previously

298 described in detail ${ }^{31}$, and its advantage is in avoiding experimental artifacts due to the

299 different velocities of embryo development. In situ hybridization results (Fig. 3B)

300 showed that both GATA5 and Nkx2.5 expression were down-regulated on the

301 imidacloprid-treated side, while VMHC and BMP2 expression was maintained. The

302 results of RT-PCR showed that imidacloprid exposure increased Wnt3a expression;

303 dramatically inhibited GATA4, TBX5, VEGFR2 and BMP4 expression, but did not

304 affect BMP2, Fgf8 and VMHC expression (Fig. 3C). The comparisons of gene

305 expressions are shown in Figure S.2A. The results of westren-blot showed that the

306 imidacloprid exposure inhibited GATA4, GATA6 and TBX5 expression at protein

307 level (Fig. 3D-E).

$308 \quad$ Imidacloprid exposure interfered with EMT at gastrula chick embryos

309 Cardiac progenitor cells derived from lateral plate mesoderm cells, which were

310 undergo EMT (Fig. 4A). During EMT, E-Cadherin down-regulation and N-cadherin

311 up-regulation are considered to be indispensable ${ }^{32}$. Here, E-Cadherin in

312 DMSO-treated embryos was mainly expressed in the apical side of epiblast (Figs. 4B, 
313 B1-B1'). In contrast, expression of E-Cadherin in imidacloprid-induced embryos

314 extended to epiblast, mesoderm and hypoblast (Figs. 4C, C1-C1'). RT-PCR showed

315 that imidacloprid treatment reduced expression of $\mathrm{N}$-cadherin and increased that of

316 E-Cadherin.

317 During chick gastrulation, the earliest sign of EMT is the breakdown of BM at the

318 midline ${ }^{33-35}$. Compared to 0.1\% DMSO-treated embryos (Figs. 4D, D1-D1'),

319 imidacloprid treatment shortened the midline distance $(\mathrm{DMSO}=241.80 \pm 13.99 \mu \mathrm{m}, \mathrm{n}$

$320=10$, imidacloprid $=170.50 \pm 7.60 \mu \mathrm{m}, \mathrm{n}=10, \mathrm{P}<0.01 ;$ Figs. 4E, E1-E1', F),

321 implying that EMT was delayed. RT-PCR data (Fig. 4G) showed no significant

322 difference between the expression of RhoA between DMSO and imidacloprid groups.

323 Imidacloprid treatment reduced the expression of P120, $\beta$-catenin, CX43 and

324 claudin12; increased the expression of Vinculin, Par3 and occluding, but had no effect

325 on expression of AJs and TJs, including Wnt3a, Claudin-1, ZO-1 and $\alpha$-actin. As a

326 result, it is concluded that imidacloprid treatment induced delayed EMT during

327 cardiogenesis in gastrula chick embryo. The comparisons of gene expressions are

328 shown in Figure S. 2B and C. The results of westren-blot showed that the expression

329 imidacloprid exposure down regulated $\mathrm{N}$-cadherin, but up regulated E-cadherin at

330 protein level (Figs. 4H-I).

$331 \quad$ Imidacloprid inhibited the migration of cardiac progenitor cells

332 Cardiac progenitor cells are the resources of the heart tube and migrate bilaterally

333 in the lateral plate mesoderm to eventully form the cardiac crescent ${ }^{9,10}$. To follow the

334 migration trajectory of cardiac progenitor cells, DiI dye was injected into anterior 
335

336

primitive streaks in $\mathrm{HH} 3$ chick embryos as shown in Figs. 5A, 5B. The embryos were then exposed and cultured on either 0.1\% DMSO (control) on both sides (Fig. 5A) or with imidacloprid on one side (Fig. 5B). The photographs were taken after 9-hour and 20-hour incubations. The results showed that the $\mathrm{Dil}^{+}$mesoderm cells in the control group migrated symmetrically at bilateral sides of embryos $(n=18, P>0.05$; Figs. 5C-E, C1-E1, F), while many fewer Dil $^{+}$mesoderm cells were observed after 9- and 20-hour incubations at the side of imidacloprid-treatment compared to the control $(\mathrm{DMSO}=91.00 \pm 1.38$, imidacloprid $=43.38 \pm 1.45, \mathrm{n}=18, \mathrm{P}<0.001 ;$ Figs. 5G-I, G1-I1, J-K).. This difference in $\mathrm{Dil}^{+}$cardiac progenitor cell migration clearly suggests that imidacloprid exposure restrained the cell migration of cardiac precursors towards the site of heart tube formation.

Imidacloprid exposure suppressed the migration, polarization, and protrusion formation of cardiac cells in vitro.

To examine the behavior of treated cells, we used H9c2 cells cultured in vitro in presence of imidacloprid. The scratch-wound assay showed that imidacloprid exposure inhibited H9c2 cells migration, as reflected in the extent of "wound" closure after $24 \mathrm{~h}$ incubation from the $0.1 \%$ DMSO and imidacloprid-treated groups respectively $(12 \mathrm{~h}: \mathrm{DMSO}=41.93 \pm 1.06 \%$, imidacloprid $=32.54 \pm 2.66 \%, \mathrm{P}<0.05$; 24h: $\mathrm{DMSO}=61.47 \pm 0.92 \%$, imidacloprid $=46.81 \pm 2.07 \%, \mathrm{P}<0.001, \mathrm{n}=8$ for each group; Figs. 6A, B, B1-B2, C, C1-C2, D). Actin and Myh7 are primary cytoskeletal components and are involved in the formation of cell filopodia, lamellipodia and protrusions during cell migration ${ }^{36}$. F-actin and Myh7 fluorescent 
357

358

359

360

361

362

363

364

365

366

367

368

369

370

371

372

373

374

375

376

377

378

microscopy demonstrated that compared to $0.1 \%$ DMSO exposure (Figs. 6E-F, I) imidacloprid exposure (Figs. 6G-H, J) caused a loss of cell polarization. To quantify this effect, the ratios of long to short axes of cells exposed to either DMSO or imidacloprid were calculated.

Elongation of cells exposed to imidacloprid was significantly less than that of 0.1\% DMSO-treated control cells $(\mathrm{DMSO}=3.13 \pm 0.24 \mu \mathrm{m}, \mathrm{n}=25$, imidacloprid $=$ $2.31 \pm 0.11 \mu \mathrm{m}, \mathrm{n}=25, \mathrm{P}<0.01 ;$ Fig. $6 \mathrm{~K})$. More cell protrusions occurred in the majority of cells exposed to $0.1 \%$ DMSO compared to those treated with imidacloprid $(\mathrm{DMSO}=85.69 \pm 3.19$, imidacloprid $=59.79 \pm 2.89, \mathrm{n}=10$ for each group, $\mathrm{P}<0.01$ Fig. 6L). In addition, the fluorescence intensities of Myh7 were determined (DMSO = $188.50 \pm 0.94, \mathrm{n}=25$, imidacloprid $=136.60 \pm 3.10, \mathrm{n}=32, \mathrm{P}<0.001 ;$ Fig.6M) RT-PCR data (Fig.6N) revealed that imidacloprid treatment reduced the expressions of Vinculin, Par3, ZO-1, CX-43, Claudin-1 and a-actin, but increased the expression of P120. The other tight junction gene (Claudin-12) was not affected. The comparisons of gene expressions are shown in Figure S.2D. Furthermore, we also detected the behavior of imidacloprid-treated chicken cardiac muscle cells ${ }^{37}$. The results confirmed that imidacloprid exposure could suppress the migration, polarization, and protrusion formation of cardiac cells in vitro (Figure S.4).

\section{Discussion}

The toxicity of imidacloprid varies greatly across species. As a neurotoxic insecticide, it has been used globally to control sucking insects in agriculture and 
379 animal husbandry ${ }^{19}$. Similarly, monocrotophos, an organophosphate insecticide, also

380 has been found to greatly affect the development of zebrafish in a

381 concentration-dependent manner ${ }^{38}$ It has been reported that concentrations of

382 imidacloprid in the environment was $320 \mu \mathrm{g} / \mathrm{L}$ near Noordwijkerhout, Nethelands,

383 exceeding European toxicity directives, while one fifth of water samples taken in

384 California were above the United States Environmental Protection Agency's level for

385 invertebrates (35 $\mu \mathrm{g} / \mathrm{L}$ for acute toxicity and $1.05 \mu \mathrm{g} / \mathrm{L}$ for chronic toxicity) ${ }^{39}$.

386 Accumulation of this pesticide on plants and animals will inevitably be transferred to

387 humans through close contacts and food contamination. A study on the biological

388 safety of imidacloprid products is therefore particularly important ${ }^{27,40}$. In a previous

389 study, we conducted a concentration gradient to select the proper concentration. In our

390 previous study, we conducted a concentration gradient to select the proper

391 concentration. We found that mortality and ratio of malformations were both

392 increased with the increase of the concentration ${ }^{41}$. The concentration of imidacloprid

$393(500 \mu \mathrm{M})$ in this study was similar to that reported for earlier literature reports ${ }^{27}$. We

394 considered that, for an acute toxicity experiment, the acceptable range should be less

395 than 1000 times the environmental concentrations, and the concentration we selected

396 here, $500 \mu \mathrm{M}(127.8 \mathrm{mg} / \mathrm{L})$, was within this range. $\alpha 7 \mathrm{nAChR}$ has been reported to be

397 increased during cardiac hypertrophy in the rat ${ }^{42}$. Our previous study also found that

398 AChR and AChE were presented in early chick embryos. We detected these

399 expressions with acetylcholinesterase and acetylcholine receptors by RT-PCR. This

400 work shows that expressions of both acetylcholinesterase and acetylcholine receptors 
were inhibited by treatment with imidacloprid ${ }^{43}$. Pregnant women is a kind of vulnerable groups, human embryonic development is likely to be affected by cumulative toxic effects if pregnant women are exposed over the long-term to imidacloprid. During embryogenesis, the heart is the first organ to be developed. Severe developmental defects in the heart could cause embryonic death. Hence, it is vital to determine whether or not exposure to this widely-used chemical could affect development.

The chick embryo was selected to systemically investigate the potential toxic effect of imidacloprid exposure on early heart tube formation in this study. Chick embryos develop to HH10 for about 2days. Ventricular segment firstly bulge ventrally and then flips to the right side. In this way, the heart fuses and a primitive C-shaped heart tube is formed ${ }^{16}$. At 4.5 days, the cardiac looping process is completed ${ }^{44}$. At 14 days, the expansion and growth of the ventricular wall has ended and a mature heart is produced (Fig. 1A). Our results show that imidacloprid exposure significantly retarded the growth of chick embryos (Fig. 1) and increased the incidence of different degrees of cardiac malformations (Fig. 2). MF20, the marker of myosin II heavy chain in muscles, was exploited to outline the morphology of heart tubes, and is clearly expressed in the myocardium of single and complete heart tubes in $0.1 \%$ DMSO-treated control (Figs. 2F, F1-F3). In contrast, the unfused cavity marked by MF20 is evident in the imidacloprid-treated group (Figs. 2I, I1-I3) implying that imidacloprid exposure might result in cardia bifida. Furthermore, the development of ventricular wall and trabecular muscle in 4.5 days was delayed by the imidacloprid 
treatment. In comparison to the reduction of cardiac volume and weight in 14 days, the thickness of right ventricular wall was significantly increased in compensation following imidacloprid exposure (Fig. 1O). Imidacloprid exposure-induced embryonic mortality in the first two days is much higher than in the other two phases (Fig. 1B). This finding also further confirms that the first two days is the crucial period for heart tube formation. It was this period that we addressed in this study.

Morphogenesis of the heart tube during embryo development relies on a precisely coordinated expression of cardiac-associated genes. Crescent formation mainly requires several signal factors, including Wnt, BMP and Fgf signaling, which coordinately control cardiomyocyte differentiation-related genes (NKX2.5, GATA4/5/6 and T-box). . Among those signal pathways, Wnt3a/ $\beta$-catenin signal is deemed to be a negative regulator, the others being positive ${ }^{16}$. In this study, we found that imidacloprid exposure up-regulated Wnt3a expression and slightly down-regulated the expression of BMP4, with not much change being observed in the expressions of BMP2 and Fgf8. Knock-out or mutation of GATA4 and GATA5, the zinc-finger transcription factors for cardiogenesis, leads to cardia bifida in mice ${ }^{45}$ whilst over-expression of GATA5 induces ectopic Nkx2.5 expression. The GATA6 promoter in both mouse and chick contains functionally important Nkx2.5 binding sites. Similarly, the murine Nkx2.5 promoter contains GATA sites that are involved in early heart field expression ${ }^{46}$. Likewise, the unlooped heart is associated with TBX5 mutation. Furthermore, VEGFR2 and its ligand VEGF are the cardiac- and endothelial marker at the cardiac crescent stage ${ }^{47}$. It has been observed that imidacloprid 
exposure could result in an obvious down-regulation of VEGFR2 (Fig. 3). From the results of western blot we also found the down regulaoted of GATA4, GATA6 and TBX5. All these results imply that imidacloprid-treated could significantly inhibit cardiomyocyte differentiation during heart tube formation.

It is known that cardiac crescent cells date from myocardial precursor cells initiated at the anterior primitive streak of gastrula embryo. Using the $\mathrm{Dil}^{+}$migration assay, we showed that the cell migration of myocardial precursor cells was suppressed by the exposure to imidacloprid (Figs. 5G-I). In comparison to the $0.1 \%$ DMSO-exposed side of embryos, the less migratory $\mathrm{Dil}^{+}$myocardial precursor cells in the imidacloprid-exposed side demonstrate that imidacloprid exposure indeed interfered with precardiac cell migration toward the primary heart fields. However, the possibility of an influence on cell proliferation cannot be excluded.

To investigate how imidacloprid affects cell migration, we employed scratch wound assay and found that exposure inhibited H9c2 cells and chicken cardiac muscle cells migration (Figs. 6B-B2, C-C2 and Fig.S4). It has been reported that cells migration properties are related to cellular cytoskeleton modulation or to relevant adherence factors 48,49 . These data show that imidacloprid exposure disturbed cell internal structure (Fig. 6G) and reduced the number of stress fibers (Fig. 6H). Moreover, cell migration also relies on cell-cell junctions, including AJs, TJs and GJs etc (Fig. 6H). Classic cadherins, including E-cadherin and $\mathrm{N}$-cadherin, are crucial molecules in calcium-dependent cell adhesion and supply trans-homophilic binding to other cadherins on adjacent cells, whereas their intracellular domains firsthand interact with 
467

468

469

470

471

472

473

474

475

476

477

478

479

480

481

482

483

484

485

486

487

488

p120-catenin. Vinculin, an actin-binding protein, connects intracellular actin filaments by forming a mixture of, for example, $\alpha$-catenin and $\beta$-catenin ${ }^{50}$. TJs located at the top of the lateral membranes, including the claudin family and occluding, exhibit "barrier" and "fence" functions that involve binding to intracellular ZO-1 ${ }^{51}$. GJs, such as $\mathrm{CX} 43$, form multiple channels that allow the passage of small molecules and electrical signals ${ }^{52}$. All the mentioned-above cell adhesion molecules were down-regulated by imidacloprid (Fig. 6N), which suggested that this exposure certainly interfered with cell migration and cardiac crescent formation during heart tube formation.

Cardiac precursor cells derive from epiblast cells after undergoing EMT. EMT not only needs to down-regulate expression of E-cadherin (required to maintain epithelial cell contact) but also requires up-regulating the expression of $\mathrm{N}$-cadherin, the mesenchymal cell adhesion molecules. The Wnt/ $\beta$-catenin signaling pathway plays regulatory role in the adhesion belt. Moreover, break-down of BM, marked by laminin and the alteration of others cell-cell adhesion factors (AJs, TJs, GJs), are also very important in EMT. In this research, imidacloprid treatment led to E-cadherin up-regulation and N-cadherin down-regulation at mRNA and protein levels in the gastrula chick embryos. This treatment also enhanced laminin expression but had little influence on AJs (p120, Vinculin, Par3, $\beta$-catenin) and GJs (CX43). These data indicate that imidacloprid-exposure interference with EMT is achieved through altering the relevant adhesion molecules.

In summary, these studies reveal that imidacloprid exposure negatively influenced 
489 EMT, cell migration and cell differentiation in heart tube formation. Figure 7

490 summarises schematically how imidacloprid might cause these changes. But, at

491 present, the mechanisms of cardiogensis are only incompletely understood.

492 Furthermore, imidacloprid products are likely to flow into drinking water in poultry

493 farms, which may have impact on the quantity and quality of hatching eggs.

494 Thus, further experiments are required to explore the precise molecular 495 mechanism by which imidacloprid affects cardiogenesis, thereby contribute to 496 improve poultry industry.

497

\section{Acknowledgements}

499 We would like to thank Dr. Thomas M. Schultheiss for the GATA5 plasmid. This 500 study was supported by an NSFC grant (31401230, 81571436), the Science and 501 Technology Planning Project of Guangdong Province (2014A020221091, 502 2014A020213008), the Science and Technology Program of Guangzhou 503 (201510010073) and Guangdong Natural Science Foundation. 2016A030311044.

504

505

506

507

508

509

510

\section{Competing Financial Interest}

The authors have declared that no competing interests exist.

Figure legends

Figure 1. Imidacloprid retarded development of the chick embryos and resulted in

abnormal heart formation. A: The illustration shows the crucial points (2-, 4.5- and 
511 14-day) in chick embryos heart development. B: Graph shows the mortality rate in

$5120.1 \%$ DMSO and $500 \mu \mathrm{M}$ imidacloprid-treated chick embryos at days 2, 4.5 and 14 ,

513 respectively. C-C2: Representative appearance of 0.1\% DMSO-treated chick embryos

514 for 0- (C), 21- (C1) and 48- (C2) hs. C3-C5: Representative appearance of

515 imidacloprid-treated chick embryos for 0- (C3), 21- (C4) and 48- (C5) hs. D: Bar

516 chart shows the length of embryos following treatment at 0-, 21-, 48h. E: Bar chart

517 shows the pair numbers of somites at $48 \mathrm{~h}$. F: Bar chart shows the whole embryo

518 weight of chick embryos in E4.5. G, G1-G2: Representative appearance of the

519 4.5-day developing hearts in 0.1\% DMSO-treated group $(\mathrm{G})$, transverse section was

520 taken at the level indicated by dotted lines in F and stained with H\&E stains (G1). The

521 high magnification images were taken from the sites indicated by boxed regions in G1

522 (G2). The black line and boxed region in G2 marked the ventricular wall and

523 trabecular muscle, respectively. H, H1-H2: The example shows the appearance of

524 4.5-day developing hearts in the imidacloprid- treated group $(\mathrm{H})$, transverse section

525 was taken at the level indicated by dotted lines in $\mathrm{H}$ and stained with H\&E stains (H1).

526 The high magnification images were taken from the sites indicated by boxed regions

527 in $\mathrm{H} 1(\mathrm{H} 2)$. The black line and boxed region in $\mathrm{H} 2$ dotted the ventricular wall and

528 trabecular muscle, respectively. I: Bar chart compares the ventricular wall thickness

529 of hearts. J: Bar chart compares the trabecular muscle layers. K: Representative

530 appearance of the 14-day mature hearts in 0.1\% DMSO-treated group. L: Example

531 shows appearance of 14-day mature hearts in the imidacloprid- treated group. K1, L1:

532 Transverse section was taken at the levels indicated by dashed lines in K and L. M-N: 
533 Bar chart shows the heart weight and the whole embryo weight. O: The bar chart

534 showing the thickness of ventricular wall in 14-day mature hearts. Abbreviations: LV,

535 left ventricle; RV, right ventricle; IVS, interventricular septum. Scale bars $=2000 \mu \mathrm{m}$

536 (C, C3); $1000 \mu \mathrm{m}(\mathrm{C} 1-\mathrm{C} 2, \mathrm{C} 4-\mathrm{C} 5) ; 500 \mu \mathrm{m}(\mathrm{G}-\mathrm{H}) ; 300 \mu \mathrm{m}(\mathrm{G} 1-\mathrm{H} 1) ; 50 \mu \mathrm{m}(\mathrm{G} 2-\mathrm{H} 2)$;

$537 \quad 300 \mu \mathrm{m}(\mathrm{K}-\mathrm{L}) ; 1000 \mu \mathrm{m}(\mathrm{K} 1-\mathrm{L} 1)$.

538

539

540

541

Figure 2. The classification of imidacloprid exposure-induced heart malformations

in gastrula chick embryos. A-A3: Representative appearances of phenotypes classification of hearts in gastrulating chick embryos immunofluorescently-stained with MF20 antibody, including normal (A), mild (A1), intermediate (A2) and severe (A3), respectively. B-B3: In situ hybridization shows VMHC expression in representative appearances of phenotypes classification of hearts in gastrulating chick embryos. C: Bar chart shows the rate of heart phenotype classification (\%) in $0.1 \%$ DMSO- and imidacloprid-treated group. D-E: Representative bright-field images of 0.1\% DMSO-treated HH10 embryo (D) and heart tube immunofluorescently-stained with MF20 antibody (E). F, F1-F3: F: Representative transverse sections at the levels indicated by dotted white line in E. DAPI staining is used as a counterstain in F1. F2 is the merged image. F3 is the enlarged view of boxed region in F2. G-H: Representative bright-field images of 0.1\% DMSO-treated HH10 embryo (G) and heart tube immunofluorescently-stained with MF20 antibody (H). I, I1-I3: I: Representative transverse sections at the levels indicated by dotted white line in $\mathrm{H}$. DAPI staining is used as a counterstain in I1. I 2 is the merged image. I 3 is the 
555

556

557

558

559

560

561

562

563

564

565

566

567

568

569

570

571

572

573

574

575

576

enlarged view of boxed region in I2. Scale bars $=150 \mu \mathrm{m}(\mathrm{A} 1-\mathrm{A} 4, \mathrm{~B} 1-\mathrm{B} 4, \mathrm{E}, \mathrm{H}) ; 500$

$\mu \mathrm{m}(\mathrm{D}, \mathrm{G}) ; 100 \mu \mathrm{m}(\mathrm{F}, \mathrm{F} 1-\mathrm{F} 3, \mathrm{I}, \mathrm{I} 1-\mathrm{I} 3)$.

Figure 3. Imidacloprid exposure repressed the differentiation of cardiac progenitor

cells. A: Overview of the signaling pathways that have been implicated into cardiomyocyte formation. B1-B4: The embryos were incubated with 0.1\% DMSO (left) and imidacloprid (right) at either side until HH7 and processed for in situ hybridization for GATA5 (B1), NKX2.5 (B2), VMHC (B3), BMP2 (B4). B1'-B4': Representative transverse sections at the levels indicated by dotted black lines in B1-B4. C: RT-PCR showing the expressions at HH7 chick embryos. D: Western-bolt showing the expressions at protein level in $\mathrm{HH} 7$ chick embryos. E: The bar chart showing the comparisons of gene expressions in D. Scale bars $=200 \mu \mathrm{m}(\mathrm{B} 1-\mathrm{B} 4)$; $100 \mu \mathrm{m}\left(\mathrm{B} 1^{\prime}-\mathrm{B} 4{ }^{\prime}\right)$.

Figure 4. Imidacloprid exposure interfered with EMT during chick gastrulation. A:

The illustration shows the EMT during chick gastrulation. B: Representative images of $0.1 \%$ DMSO-treated HH4 chick embryos immunofluorescently-stained with E-Cadherin. B1-B1': The transverse sections at the levels indicated by dotted white line in B. The section was counterstained with DAPI (B1'). E-Cadherin is expressed on the apical side of epiblast of $0.1 \%$ DMSO-treated embryo (white arrow in B1'). C: Representative images of imidacloprid-treated HH4 chick embryos immunofluorescently-stained with E-Cadherin. C1-C1': The transverse sections at 
577 levels indicated by dotted white line in C. The section was counterstained with DAPI

578 (C1'). E-Cadherin expression level was enhanced on epiblast layer, and ectopic 579 expression in the mesoderm layer following imidacloprid treatment (white arrows in

580 C1'). D: Representative image of $0.1 \%$ DMSO-treated HH4 chick embryos 581 immunofluorescently-stained for laminin. D1-D1': The transverse sections at levels 582 indicated by dotted white line in D. The section was counterstained with DAPI (D1'). 583 Laminin is expressed on the BM of $0.1 \%$ DMSO-treated embryo (white dotted line 584 showing the gap in D1'). E: Representative image of imidacloprid-treated HH4 chick 585 embryos immunofluorescently-stained for laminin. E1-E1': The transverse sections at 586 the levels indicated by dotted white line in E. The section was counterstained with 587 DAPI (E1'). Laminin is expressed on the BM of imidacloprid-treated embryo (white 588 dotted line showing the gap in E1'). F: Bar chart shows the gap distance of laminin $589(\mu \mathrm{m})$ with $0.1 \%$ DMSO- and imidacloprid-treated HH4 chick embryos. G: RT-PCR 590 shows the expressions $\mathrm{N}$-cadherinat mRNA level in the HH4 chick embryos. H: 591 Western-bolt showing the expressions at protein level in HH4 chick embryos. I: The 592 bar chart showing the comparisons of gene expressions in H. Scale bars $=300 \mu \mathrm{m}$

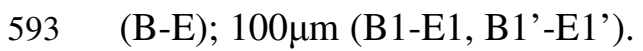
treatment on the both sides of embryos. B: The pattern of DiI-labeled cardiac 
599

600

601

602

603

604

605

606

607

610

611

612

613

614

615

616

617

618

619

620

imidacloprid exposure at right side of embryos. C-E: Fluorescence images were taken at 0- (B), 9- (C) and 20- (D) hour. Note: both sides of embryos were exposed to $0.1 \%$

DMSO. C1-E1: The merged images of bright-field and B-D respectively. F: Bar chart shows the number of cardiac precursor cells migration based on A. G-I: Fluorescence images were taken at 0- (G), 9- (H) and 20- (I) of incubation. The left sides of embryos were exposed to $0.1 \%$ DMSO, while the right sides were exposed to imidacloprid. G1-I1: The merged images of bright-field and G-I respectively. J: Bar chart shows the number of cardiac precursor cells migration based on F. K: Bar chart shows the number of embryo incidence of symmetrical migration or asymmetric migration in $0.1 \%$ DMSO- and imidacloprid groups. Scale bars $=600 \mu \mathrm{m}(\mathrm{C}-\mathrm{E}, \mathrm{C} 1-\mathrm{E} 1$, G-I, G1-I1).

Figure 6. The imidacloprid exposure suppressed H9c2 cells migration, polarization and protrusion formation. A: The sketch illustrates migration of $\mathrm{H} 9 \mathrm{c} 2$ cells as detected by the wound-healing assay. B-C: The representative images of H9c2 cells scratch test at 0-hour incubation from $0.1 \%$ DMSO-treated (B) and imidacloprid-treated (C) groups respectively. B1-C1, B2-C2: The representative images of H9c2 cells scratch test at 12-hour (B1, C1), 24-hour (B2, C2). D: The bar chart shows the percentage of wound closure (\%) at 12-hour, 24-hour. E-F: Representative image of actin filaments in $0.1 \%$ DMSO -treated H9c2 cells were visualized by staining with F-actin (red), and cell nuclei were stained with DAPI (blue). White dotted lines show the long and short axes of cells. F is the enlarged view 
621 of $\mathrm{E}$. (The boxed region in $\mathrm{F}$ shows stress fiber assay in H9c2 cells). G-H:

622 Representative image of actin filaments in imidacloprid-treated H9c2 cells were 623 visualized by staining with F-actin (red), and cell nuclei were stained with DAPI 624 (blue). White dotted lines show the long and short axes of cells. $\mathrm{H}$ is the enlarged 625 view of $\mathrm{G}$. (The boxed region in $\mathrm{H}$ shows stress fiber assay in H9c2 cells). I-J: 626 Representative images of $0.1 \%$ DMSO and imidacloprid-treated H9c2 cells 627 immunofluorescently-stained with Myh7, respectively. K: Bar chart showing the ratio 628 of long axis to short axis. $\mathbf{L}$ : Bar chart shows cells containing stress fibers (\%). $\mathbf{M}$ : 629 Bar chart shows fluorescence intensity of Myh7 (AU). N: RT-PCR showing the 630 expressions at mRNA level in $\mathrm{HH} 7$ chick embryos exposed either $0.1 \%$ DMSO or 631 imidacloprid. Scale bars $=200 \mu \mathrm{m}(B, B 1-B 2, C, C 1-C 2) ; 100 \mu \mathrm{m}($ E- J).

632

634

635

636

637

638

639

640

641

642

\section{5}

Figure 7. Model depicting how imidacloprid exposure induced heart tube malformation during chick cardiogenesis.

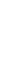


643

644

645

646

647

648

649

650

651

652

653

654

655

656

657

658

659

660

661

662

663

664

1. Ray, H. J.; Niswander, L., Mechanisms of tissue fusion during development. Development 2012, 139, 1701-1711.

2. Alsan, B. H.; Schultheiss, T. M., Regulation of avian cardiogenesis by Fgf8 signaling. Development 2002, 129, 1935-1943.

3. Jin, Y. M.; Wang, G.; Zhang, N.; Wei, Y. F.; Li, S.; Chen, Y. P.; Chuai, M.; Lee, H. S.; Hocher, B.; Yang, X., Changes in the osmolarity of the embryonic microenvironment induce neural tube defects. Mol Reprod Dev 2015, 82, 365-376.

4. Li, S.; Wang, G.; Gao, L. R.; Lu, W. H.; Wang, X. Y.; Chuai, M. L.; Lee, K. K. H.; Cao, L.; Yang, X. S., Autophagy is involved in ethanol-induced cardia bifida during chick cardiogenesis. Cell Cycle 2015, 14, 3306-3317.

5. Martinsen, B. J., Reference guide to the stages of chick heart embryology. Dev Dyn 2005, 233, 1217-1237.

6. Waldo, K. L.; Kumiski, D. H.; Wallis, K. T.; Stadt, H. A.; Hutson, M. R.; Platt, D. H.; Kirby, M. L., Conotruncal myocardium arises from a secondary heart field. Development 2001, 128, 3179-3188.

7. Nakaya, Y.; Sheng, G. J., An amicable separation Chick's way of doing EMT. Cell Adh Migr 2009, 3, 160-163.

8. Hay, E. D., The mesenchymal cell, its role in the embryo, and the remarkable signaling mechanisms that create it. Dev Dyn 2005, 233, 706-720.

9. Yang, X. S.; Chrisman, H.; Weijer, C. J., PDGF signalling controls the migration of mesoderm cells during chick gastrulation by regulating $\mathrm{N}$-cadherin expression. Development 2008, 135, 3521-3530. 
665

666

667

668

669

670

671

672

673

674

675

676

677

678

679

680

681

682

683

684

685

686

10. Yang, X. S.; Dormann, D.; Munsterberg, A. E.; Weijer, C. J., Cell movement patterns during gastrulation in the chick are controlled by chemotaxis mediated by positive and negative FGF4 and FGF8. Dev Cell 2002, 3, 425-437.

11. Yue, Q.; Wagstaff, L.; Yang, X.; Weijer, C.; Munsterberg, A., Wnt3a-mediated chemorepulsion controls movement patterns of cardiac progenitors and requires RhoA function. Development 2008, 135, 1029-1037.

12. Evans, S. M.; Yelon, D.; Conlon, F. L.; Kirby, M. L., Myocardial Lineage Development. Circ Res 2010, 107, 1428-1444.

13. Merav Beiman; Ben-Zion Shilo; Volk, T., Heartless, a Drosophila FGF receptor homolog, is essential for cell migration and establishment of several mesodermal lineages. Genes Dev 1996, 10, 2993-3002.

14. Nakajima, Y.; Sakabe, M.; Matsui, H.; Sakata, H.; Yanagawa, N.; Yamagishi, T., Heart development before beating. Anat Sci Int 2009, 84, 67-76.

15. Schultheiss, T. M.; Xydas, S.; Lassar, A. B., Induction of avian cardiac myogenesis by anterior endoderm. Development 1995, 121, 4203-4214.

16. Brand, T., Heart development: molecular insights into cardiac specification and early morphogenesis. Dev Biol 2003, 258, 1-19.

17. Pradhan, L.; Gopal, S.; Li, S.; Ashur, S.; Suryanarayanan, S.; Kasahara, H.; Nam, H. J., Intermolecular Interactions of Cardiac Transcription Factors NKX2.5 and TBX5. Biochemistry 2016, 55, 1702-1710.

18. Marinaccio, C.; Nico, B.; Ribatti, D., Differential expression of angiogenic and anti-angiogenic molecules in the chick embryo chorioallantoic membrane and selected 
687

688

689

690

691

692

693

694

695

696

697

698

699

700

701

702

703

704

705

706

707

708

organs during embryonic development. Int J Dev Biol 2013, 57, 907-916.

19. Kilpatrick, A. L.; Hagerty, A. M.; Turnipseed, S. G.; Sullivan, M. J.; Bridges, W.

C., Jr., Activity of selected neonicotinoids and dicrotophos on nontarget arthropods in cotton: implications in insect management. J Econ Entomol 2005, 98, 814-820.

20. Rust, M. K.; Denholm, I.; Dryden, M. W.; Payne, P.; Blagburn, B. L.; Jacobs, D.

E.; Bond, R.; Mencke, N.; Schroeder, I.; Weston, S.; Vaughn, M.; Coleman, G.; Kopp, S., Large-scale monitoring of imidacloprid susceptibility in the cat flea, Ctenocephalides felis. Med Vet Entomol 2011, 25, 1-6.

21. Rust, M. K.; Waggoner, M.; Hinkle, N. C.; Mencke, N.; Hansen, O.; Vaughn, M.;

Dryden, M. W.; Payne, P.; Blagburn, B. L.; Jacobs, D. E.; Bach, T.; Bledsoe, D.;

Hopkins, T.; Mehlhorn, H.; Denholm, I., Development of a larval bioassay for susceptibility of cat fleas (Siphonaptera: Pulicidae) to imidacloprid. J Med Entomol 2002, 39, 671-674.

22. Tan, Y.; Biondi, A.; Desneux, N.; Gao, X. W., Assessment of physiological sublethal effects of imidacloprid on the mirid bug Apolygus lucorum (Meyer-Dur). Ecotoxicology 2012, 21, 1989-1997.

23. He, Y.; Zhao, J.; Zheng, Y.; Desneux, N.; Wu, K., Lethal effect of imidacloprid on the coccinellid predator Serangium japonicum and sublethal effects on predator voracity and on functional response to the whitefly Bemisia tabaci. Ecotoxicology 2012, 21, 1291-1300.

24. Sillapawattana, P.; Schaffer, A., Effects of imidacloprid on detoxifying enzyme glutathione S-transferase on Folsomia candida (Collembola). Environ Sci Pollut Res 
Int 2016. DOI: $10.1007 / \mathrm{s} 11356-016-6686-1$

25. Tomizawa, M.; Casida, J. E., Neonicotinoid insecticide toxicology: mechanisms of selective action. Annu Rev Pharmacol Toxicol 2005, 45, 247-268.

26. Datar, S. P.; Bhonde, R. R., Modeling chick to assess diabetes pathogenesis and treatment. Rev Diabet Stud 2011, 8, 245-253.

27. Gu, Y. H.; Li, Y.; Huang, X. F.; Zheng, J. F.; Yang, J.; Diao, H.; Yuan, Y.; Xu, Y.; Liu, M.; Shi, H. J.; Xu, W. P., Reproductive effects of two neonicotinoid insecticides on mouse sperm function and early embryonic development in vitro. PloS one 2013, 8 , e70112.

28. Henrique, D.; Adam, J.; Myat, A.; Chitnis, A.; Lewis, J.; Ish-Horowicz, D., Expression of a Delta homologue in prospective neurons in the chick. Nature 1995, $375,787-790$.

29. Li, Y.; Wang, X. Y.; Zhang, Z. L.; Cheng, X.; Li, X. D.; Chuai, M.; Lee, K. K.; Kurihara, H.; Yang, X., Excess ROS induced by AAPH causes myocardial hypertrophy in the developing chick embryo. Int J Cardiol 2014, 176, 62-73.

30. Kamei, C. N.; Kempf, H.; Yelin, R.; Daoud, G.; James, R. G.; Lassar, A. B.; Tabin, C. J.; Schultheiss, T. M., Promotion of avian endothelial cell differentiation by GATA transcription factors. Dev Biol 2011, 353, 29-37.

31. Li, Y.; Wang, X. Y.; Wu, T.; Chuai, M. L.; Lee, K. K. H.; Wang, L. J.; Yang, X. S., PTEN is involved in modulation of vasculogenesis in early chick embryos. Biology Open 2013, 2, 587-595.

32. Takeichi, M., Self-Organization of Animal Tissues: Cadherin-Mediated Processes. 
731

732

733

734

735

736

737

738

739

740

741

742

743

744

745

746

747

748

749

750

751

752

Dev Cell 2011, 21, 24-26.

33. Sanders, E. J.; Prasad, S., Epithelial and basement membrane responses to chick embryo primitive streak grafts. Cell Differ 1986, 18, 233-242.

34. Nakaya, Y.; Sukowati, E. W.; Wu, Y.; Sheng, G., RhoA and microtubule dynamics control cell-basement membrane interaction in EMT during gastrulation. Nat Cell Biol 2008, 10, 765-775.

35. Trelstad, R. L.; Hay, E. D.; Revel, J. D., Cell contact during early morphogenesis in the chick embryo. Dev Biol 1967, 16, 78-106.

36. Lamalice, L.; Le Boeuf, F.; Huot, J., Endothelial cell migration during angiogenesis. Circ Res 2007, 100, 782-794.

37. Jin, Y. M.; Zhao, S. Z.; Zhang, Z. L.; Chen, Y.; Cheng, X.; Chuai, M.; Liu, G. S.;

Lee, K. K.; Yang, X., High glucose level induces cardiovascular dysplasia during early embryo development. Exp Clin Endocrinol Diabetes 2013, 121, 448-454.

38. Pamanji, R.; Bethu, M. S.; Yashwanth, B.; Leelavathi, S.; Rao, J. V., Developmental toxic effects of monocrotophos, an organophosphorous pesticide, on zebrafish (Danio rerio) embryos. Environ Sci Pollut Res Int 2015, 22, 7744-7753.

39. Van Dijk, T. C.; Van Staalduinen, M. A.; Van der Sluijs, J. P., Macro-invertebrate decline in surface water polluted with imidacloprid. PloS one 2013, 8, e62374.

40. Elbert, A.; Haas, M.; Springer, B.; Thielert, W.; Nauen, R., Applied aspects of neonicotinoid uses in crop protection. Pest Manag Sci 2008, 64, 1099-1105.

41. Liu, M.; Wang, G.; Zhang, S. Y.; Zhong, S.; Qi, G. L.; Wang, C. J.; Chuai, M.; Lee, K. K.; Lu, D. X.; Yang, X., From the Cover: Exposing Imidacloprid Interferes With 
753 Neurogenesis Through Impacting on Chick Neural Tube Cell Survival. Toxicol Sci

754 2016, 153, 137-148.

755 42. Ni, M.; Yang, Z. W.; Li, D. J.; Li, Q.; Zhang, S. H.; Su, D. F.; Xie, H. H.; Shen, F.

756 M., A potential role of alpha-7 nicotinic acetylcholine receptor in cardiac angiogenesis

757 in a pressure-overload rat model. J Pharmacol Sci 2010, 114, 311-319.

758 43. Wang, C. J.; Wang, G.; Wang, X. Y.; Liu, M.; Chuai, M.; Lee, K. K.; He, X. S.; Lu,

759 D. X.; Yang, X., Imidacloprid Exposure Suppresses Neural Crest Cells Generation

760 during Early Chick Embryo Development. J Agric Food Chem 2016, 64, 4705-4715.

761 44. Manner, J., Cardiac looping in the chick embryo: a morphological review with

762 special reference to terminological and biomechanical aspects of the looping process.

763 Anat Rec 2000, 259, 248-262.

764 45. Zhang, H.; Toyofuku, T.; Kamei, J.; Hori, M., GATA-4 regulates cardiac 765 morphogenesis through transactivation of the N-cadherin gene. Biochem Biophys Res 766 Commun 2003, 312, 1033-1038.

767 46. Molkentin, J. D.; Antos, C.; Mercer, B.; Taigen, T.; Miano, J. M.; Olson, E. N., 768 Direct activation of a GATA6 cardiac enhancer by Nkx2.5: evidence for a reinforcing 769 regulatory network of Nkx2.5 and GATA transcription factors in the developing heart. 770 Dev Biol 2000, 217, 301-309.

771 47. Milgrom-Hoffman, M.; Harrelson, Z.; Ferrara, N.; Zelzer, E.; Evans, S. M.; 772 Tzahor, E., The heart endocardium is derived from vascular endothelial progenitors. 773 Development 2011, 138, 4777-4787.

774 48. Dupre, L.; Houmadi, R.; Tang, C.; Rey-Barroso, J., T Lymphocyte Migration: An 
775 Action Movie Starring the Actin and Associated Actors. Front Immunol 2015, 6, 586.

776 49. Kardash, E.; Reichman-Fried, M.; Maitre, J. L.; Boldajipour, B.; Papusheva, E.;

777 Messerschmidt, E. M.; Heisenberg, C. P.; Raz, E., A role for Rho GTPases and

778 cell-cell adhesion in single-cell motility in vivo. Nat Cell Biol 2010, 12, 47-53; sup pp

$779 \quad 1-11$.

780 50. Kawauchi, T., Cell adhesion and its endocytic regulation in cell migration during

781 neural development and cancer metastasis. Int J Mol Sci 2012, 13, 4564-4590.

782 51. Ebnet, K.; Suzuki, A.; Ohno, S.; Vestweber, D., Junctional adhesion molecules

783 (JAMs): more molecules with dual functions? J Cell Sci 2004, 117, 19-29.

784 52. Laird, D. W., The gap junction proteome and its relationship to disease. Trends

785 Cell Biol 2010, 20, 92-101.

786

787 


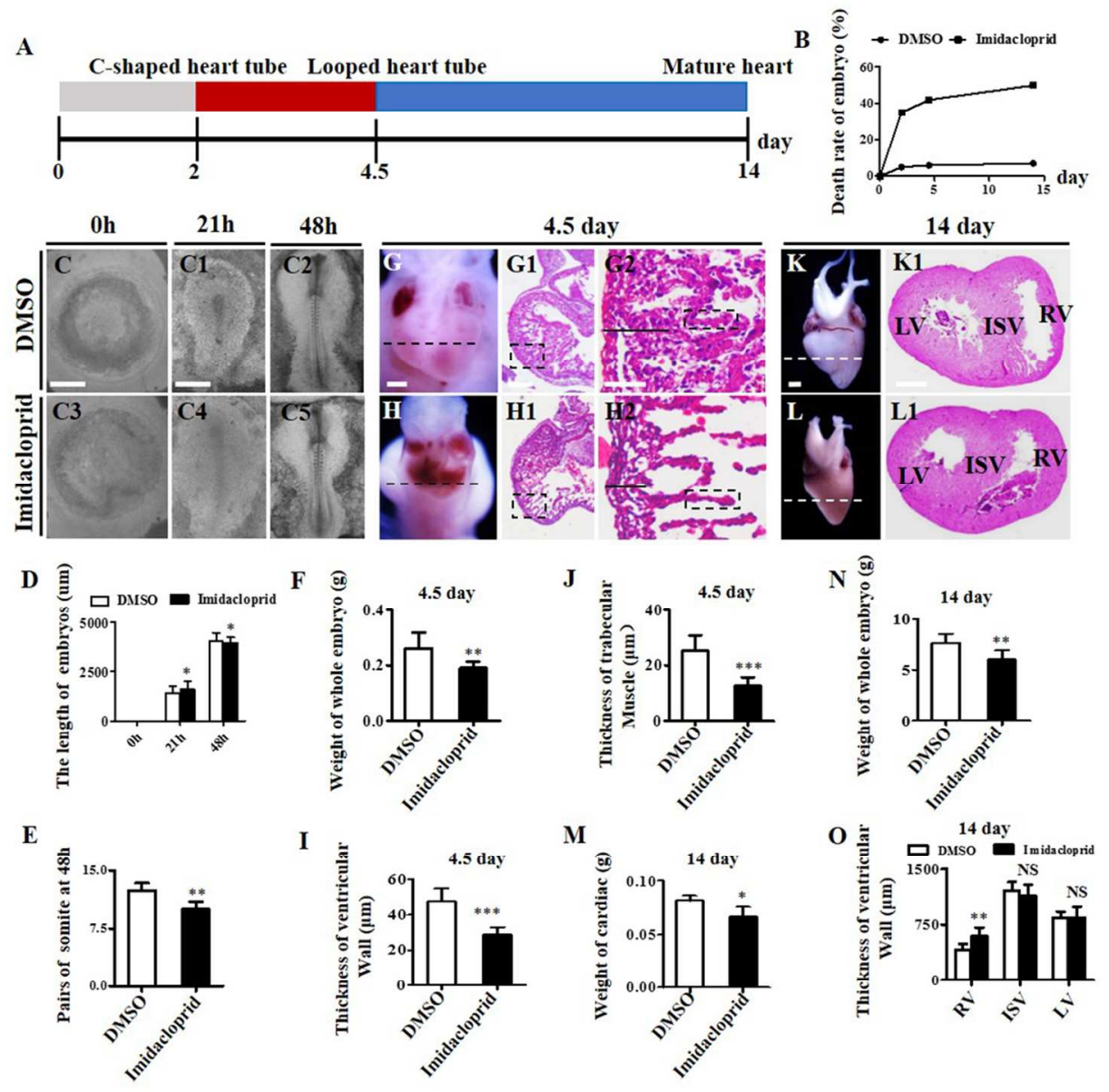

Figure 1. Imidacloprid retarded development of the chick embryos and resulted in abnormal heart formation.

A: The illustration shows the crucial points (2-, 4.5- and 14-day) in chick embryos heart development. B:

Graph shows the mortality rate in $0.1 \%$ DMSO and $500 \mu \mathrm{M}$ imidacloprid-treated chick embryos at days $2,4.5$ and 14, respectively. C-C2: Representative appearance of $0.1 \%$ DMSO-treated chick embryos for 0- (C), 21-

(C1) and 48- (C2) hs. The embryos reached to HH10 with a C-shaped heart tube after 48 hours. C3-C5:

Representative appearance of imidacloprid-treated chick embryos for 0- (C3), 21- (C4) and 48- (C5) hs. D:

Bar chart shows the pair numbers of somites at $48 \mathrm{~h}$ cultured with $0.1 \%$ DMSO and imidacloprid. E: Bar chart shows the length of $0.1 \%$ DMSO- and imidacloprid-treated embryos following treatment at 0-, 21-, 48h. F, F1-F2: Representative appearance of the 4.5-day developing hearts in $0.1 \%$ DMSO-treated group $(F)$, transverse section was taken at the level indicated by dotted lines in F and stained with H\&E stains (F1). The high magnification images were taken from the sites indicated by boxed regions in F1 (F2). The black line and boxed region in F2 marked the ventricular wall and trabecular muscle, respectively. G, G1-G2: The example shows the appearance of 4.5-day developing hearts in the imidacloprid- treated group (G), transverse section was taken at the level indicated by dotted lines in G and stained with H\&E stains (G1). The high magnification images were taken from the sites indicated by boxed regions in G1 (G2). The black line and boxed region in $\mathrm{G} 2$ dotted the ventricular wall and trabecular muscle, respectively. $\mathrm{H}$ : Bar chart shows the whole embryo weight of the $0.1 \%$ DMSO- and imidacloprid-treated chick embryos in E4.5. I: Bar chart compares the ventricular wall thickness of $0.1 \%$ DMSO- and imidacloprid- treated hearts. J: Bar chart compares the trabecular muscle layers. K: Representative appearance of the 14-day mature hearts in $0.1 \%$ DMSO-treated group. L: Example shows appearance of 14-day mature hearts in the imidacloprid- treated group. K1, L1: Transverse section was taken at the levels indicated by dashed lines in K and L. M-N: Bar chart shows the whole embryo weight and the heart weight in the 14 -day $0.1 \%$ DMSO- and imidaclopridtreated chick embryos. O: The bar chart showing the thickness of ventricular wall in 14-day mature hearts. 
Abbreviations: LV, left ventricle; RV, right ventricle; IVS, interventricular septum. Scale bars $=2000 \mu \mathrm{m}$ (C1

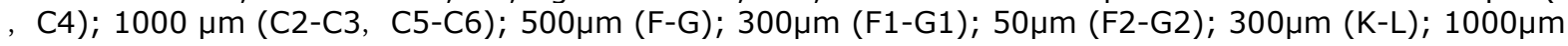
(K1-L1).

$102 \times 96 \mathrm{~mm}(300 \times 300 \mathrm{DPI})$ 

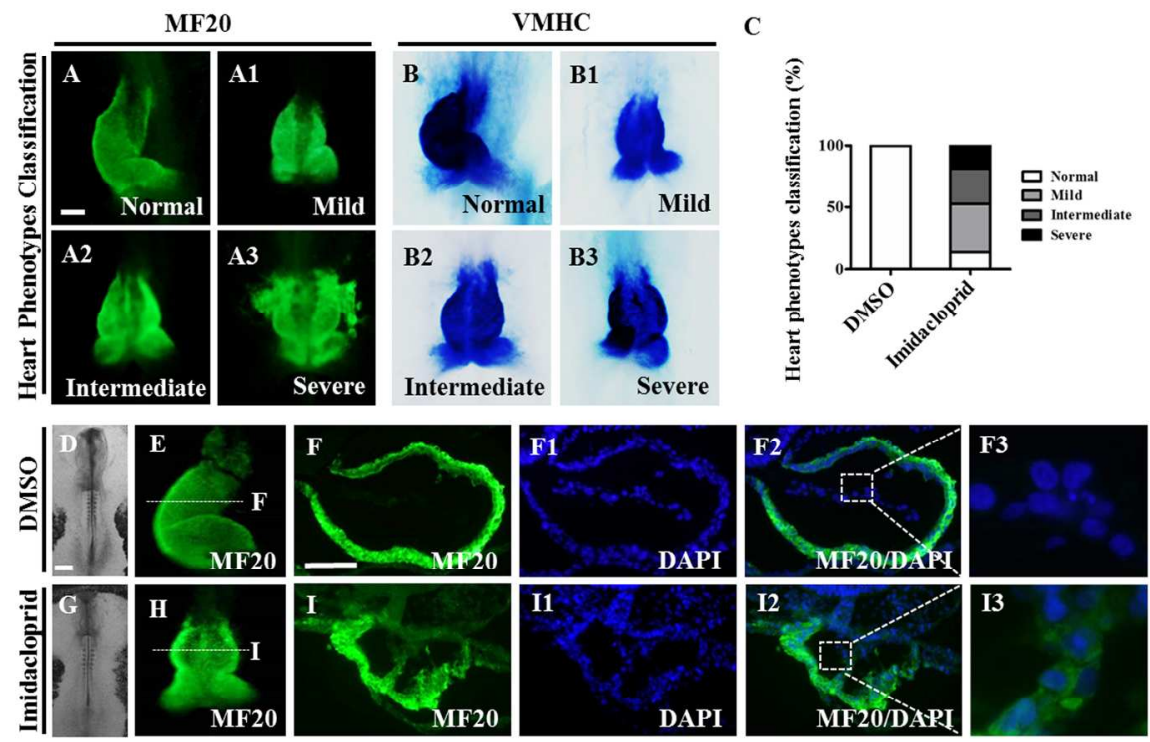

Figure 2. The classification of imidacloprid exposure-induced heart malformations in gastrula chick embryos.

A-A3: Representative appearances of phenotypes classification of hearts in gastrulating chick embryos immunofluorescently-stained with MF20 antibody, including normal (A), mild (A1), intermediate (A2) and severe (A3), respectively. B-B3: In situ hybridization shows VMHC expression in representative appearances

of phenotypes classification of hearts in gastrulating chick embryos, including normal (B), mild (B1),

intermediate (B2) and severe (B3), respectively. C: Bar chart shows the rate of heart phenotype classification (\%) in $0.1 \%$ DMSO- and imidacloprid-treated group. D-E: Representative bright-field images of $0.1 \%$ DMSO-treated HH10 embryo (D) and heart tube immunofluorescently-stained with MF20 antibody (E).

F, F1-F3: F: Representative transverse sections at the levels indicated by dotted white line in E. DAPI staining is used as a counterstain in F1. F2 is the merged image of bright-field and DAPI staining. F3 is the enlarged view of boxed region in F2. G-H: Representative bright-field images of $0.1 \%$ DMSO-treated $\mathrm{HH} 10$ embryo (G) and heart tube immunofluorescently-stained with MF20 antibody (H). I, I1-I3: I: Representative transverse sections at the levels indicated by dotted white line in H. DAPI staining is used as a counterstain in I1. I2 is the merged image of bright-field and DAPI staining. I3 is the enlarged view of boxed region in I2. Scale bars $=150 \mu \mathrm{m}(\mathrm{A} 1-\mathrm{A} 4, \mathrm{~B} 1-\mathrm{B} 4, \mathrm{E}, \mathrm{H}) ; 500 \mu \mathrm{m}(\mathrm{D}, \mathrm{G}) ; 100 \mu \mathrm{m}(\mathrm{F}, \mathrm{F} 1-\mathrm{F} 3, \mathrm{I}, \mathrm{I} 1-\mathrm{I3})$. 
A
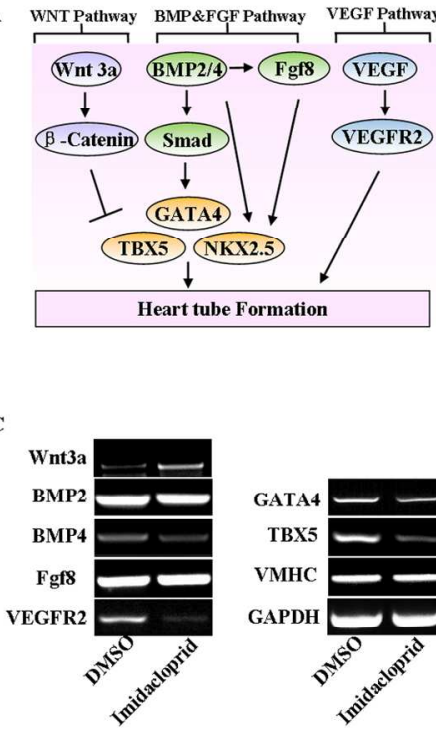

B
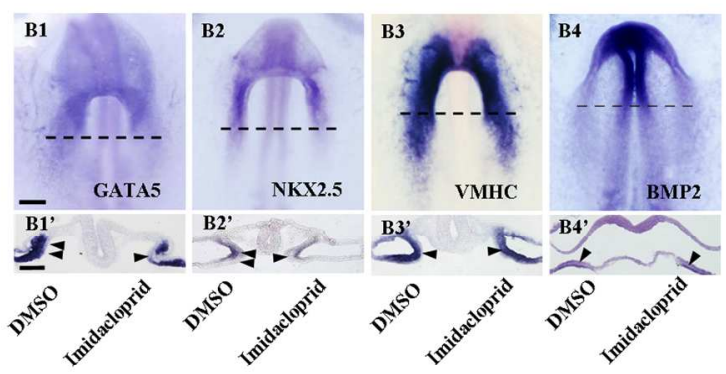

D

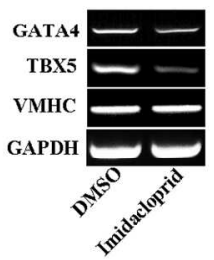

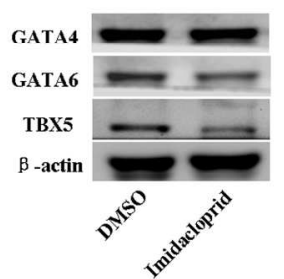

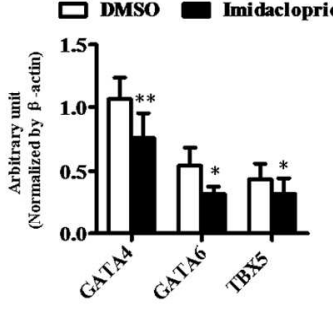

Figure 3. Imidacloprid exposure repressed the differentiation of cardiac progenitor cells. A: Overview of the signaling pathways that have been implicated into cardiomyocyte formation. B1-B4: The embryos were incubated with $0.1 \%$ DMSO (left) and imidacloprid (right) at either side until $\mathrm{HH} 7$ and processed for in situ hybridization for GATA5 (B1), NKX2.5 (B2), VMHC (B3), BMP2 (B4). B1'-B4': Representative transverse sections at the levels indicated by dotted black lines in B1-B4. C: RT-PCR showing the expressions at HH7 chick embryos. D: Western-bolt showing the expressions at protein level in HH7 chick embryos. E: The bar chart showing the comparisons of gene expressions in D. Scale bars $=200 \mu \mathrm{m}$ (B1-B4); $100 \mu \mathrm{m}\left(B 1^{\prime}-B 4^{\prime}\right)$.

$150 \times 89 \mathrm{~mm}(300 \times 300$ DPI $)$ 

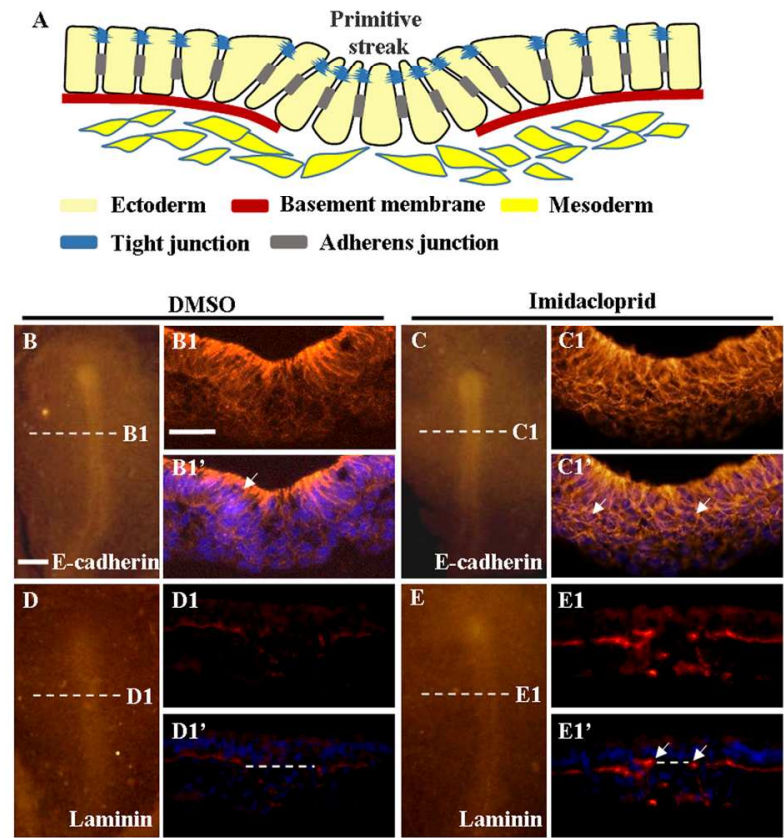

F

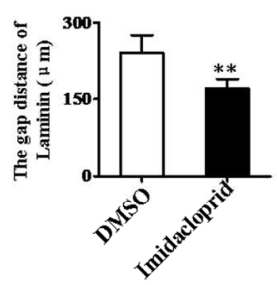

H

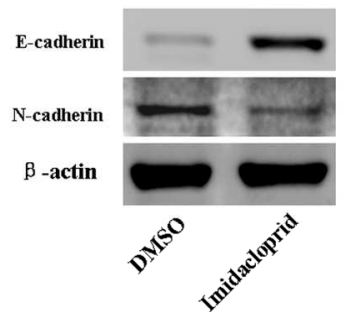

G

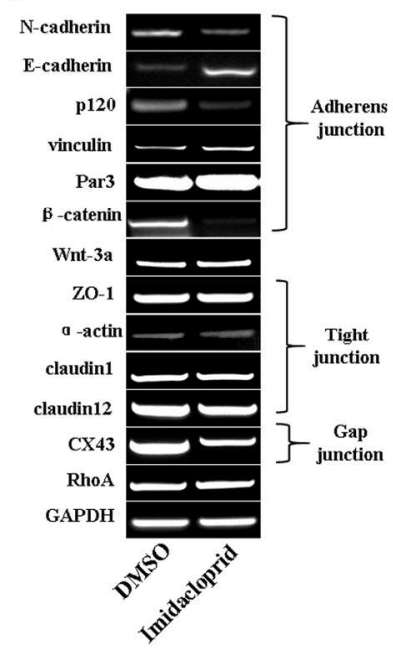

I

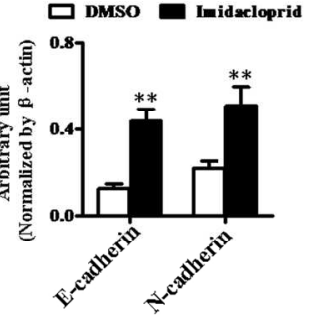

Figure 4. Imidacloprid exposure interfered with EMT during chick gastrulation. A: The illustration shows the EMT during chick gastrulation. B: Representative images of $0.1 \%$ DMSO-treated HH4 chick embryos immunofluorescently-stained with E-Cadherin. B1-B1': The transverse sections at the levels indicated by dotted white line in B. The section was counterstained with DAPI (B1'). E-Cadherin is expressed on the apical side of epiblast of $0.1 \%$ DMSO-treated embryo (white arrow in $\mathrm{B}^{\prime}$ ). C: Representative images of imidacloprid-treated $\mathrm{HH} 4$ chick embryos immunofluorescently-stained with E-Cadherin. C1-C1': The transverse sections at levels indicated by dotted white line in C. The section was counterstained with DAPI $\left(\mathrm{C} 1^{\prime}\right)$. E-Cadherin expression level was enhanced on epiblast layer, and ectopic expression in the mesoderm layer following imidacloprid treatment (white arrows in $\mathrm{C}^{\prime}$ ). D: Representative image of $0.1 \%$ DMSOtreated HH4 chick embryos immunofluorescently-stained for laminin. D1-D1': The transverse sections at levels indicated by dotted white line in D. The section was counterstained with DAPI (D1'). Laminin is expressed on the BM of $0.1 \%$ DMSO-treated embryo (white dotted line showing the gap in $D 1^{\prime}$ ). E: Representative image of imidacloprid-treated HH4 chick embryos immunofluorescently-stained for laminin.

E1-E1': The transverse sections at the levels indicated by dotted white line in $\mathrm{E}$. The section was counterstained with DAPI $\left(E 1^{\prime}\right)$. Laminin is expressed on the BM of imidacloprid-treated embryo (white dotted line showing the gap in E1'). F: Bar chart shows the gap distance of laminin $(\mu \mathrm{m})$ with $0.1 \%$ DMSOand imidacloprid-treated HH4 chick embryos. G: RT-PCR shows the expressions N-cadherinat mRNA level in the HH4 chick embryos. $\mathrm{H}$ : Western-bolt showing the expressions at protein level in $\mathrm{HH} 4$ chick embryos. I: The bar chart showing the comparisons of gene expressions in $\mathrm{H}$. Scale bars $=300 \mu \mathrm{m}(\mathrm{B}-\mathrm{E}) ; 100 \mu \mathrm{m}$ (B1-

$$
\left.E 1, B 1^{\prime}-E 1^{\prime}\right) \text {. }
$$


$150 \times 149 \mathrm{~mm}(300 \times 300 \mathrm{DPI})$ 


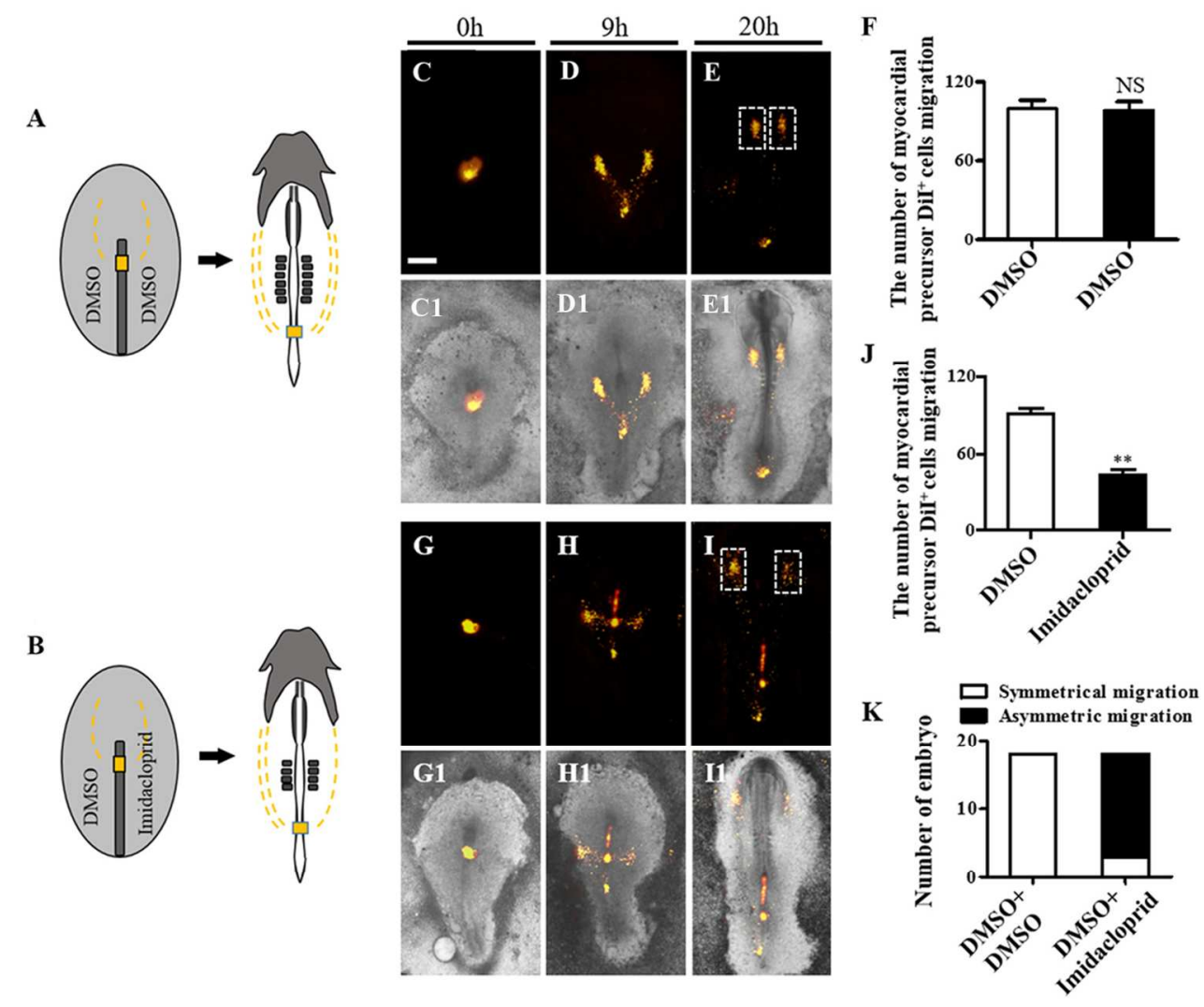

Figure 5. Imidacloprid exposure restricted cardiac progenitor cell migration. A: The pattern of DiI-labeled cardiac progenitor cell migration following $0.1 \%$ DMSO treatment on the both sides of embryos. B-D: Fluorescence images were taken at 0- (B), 9- (C) and 20- (D) hour after DiI was injected in anterior primitive streak. Note: both sides of embryos were exposed to $0.1 \%$ DMSO. B1-D1: The merged images of bright-field and B-D respectively. E: Bar chart shows the number of cardiac precursor cells migration based on A. F: The pattern of DiI-labeled cardiac progenitor cell migration following $0.1 \%$ DMSO treatment at the left side and imidacloprid exposure at right side of embryos. G-I: Fluorescence images were taken at 0- (G), 9- $(\mathrm{H})$ and 20- (I) of incubation after DiI was injected in anterior primitive streak.. The left sides of embryos were exposed to $0.1 \%$ DMSO, while the right sides were exposed to imidacloprid. G1-I1: The merged images of bright-field and G-I respectively. J: Bar chart shows the number of cardiac precursor cells migration based on F. K: Bar chart shows the number of embryo incidence of symmetrical migration or asymmetric migration in 0.1\% DMSO- and imidacloprid groups. Scale bars $=600 \mu m$ (B-D, B1-D1, G-I, G1I1).

$150 \times 124 \mathrm{~mm}(300 \times 300 \mathrm{DPI})$ 
A
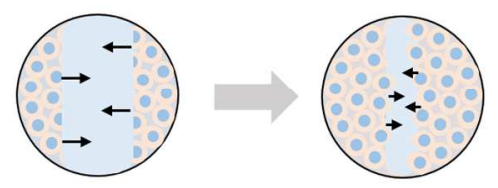

oh
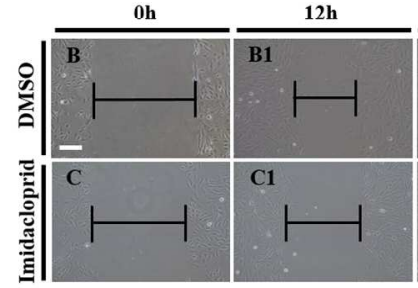

F-actin/DAPI

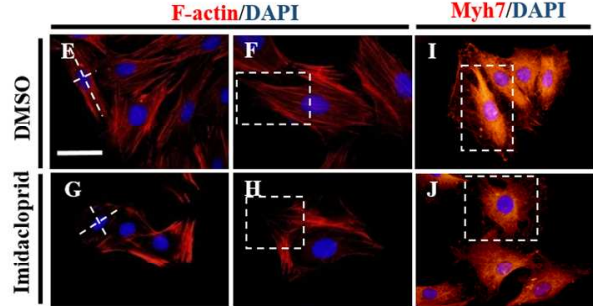

D

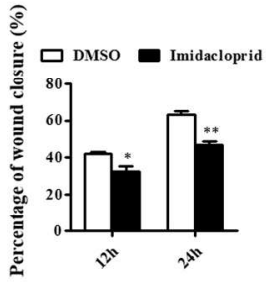

$\mathbf{K}$
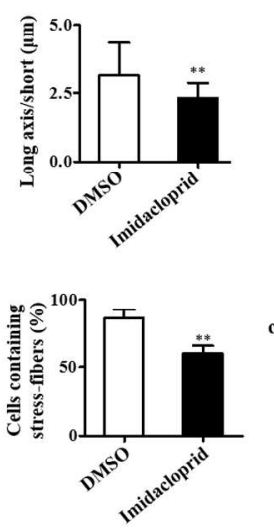

M

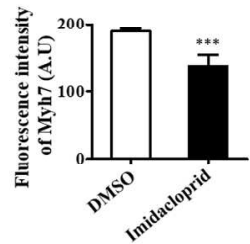

$\mathbf{N}$

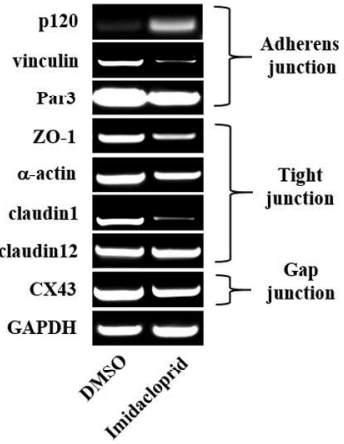

Figure 6. The imidacloprid exposure suppressed H9c2 cells migration, polarization and protrusion formation.

A: The sketch illustrates migration of $\mathrm{H} 9 \mathrm{c} 2$ cells as detected by the wound-healing assay. B-C: The representative images of $\mathrm{H} 9 \mathrm{c} 2$ cells scratch test at 0 -hour incubation from $0.1 \%$ DMSO-treated $(B)$ and imidacloprid-treated (C) groups respectively. B1-C1, B2-C2: The representative images of H9c2 cells scratch test at 12-hour (B1, C1), 24-hour (B2, C2) incubation from 0.1\% DMSO-treated (B1-B2) and imidaclopridtreated (C1-C2) groups respectively. D: The bar chart shows the percentage of wound closure (\%) at 12-

hour, 24-hour. E-F: Representative image of actin filaments in imidacloprid-treated H9c2 cells were visualized by staining with F-actin (red), and cell nuclei were stained with DAPI (blue). White dotted lines show the long and short axes of cells. $F$ is the enlarged view of $E$. (The boxed region in F shows stress fiber assay in $\mathrm{H} 9 \mathrm{c} 2$ cells). G-H: Representative image of actin filaments in imidacloprid-treated $\mathrm{H} 9 \mathrm{c} 2$ cells were visualized by staining with F-actin (red), and cell nuclei were stained with DAPI (blue). White dotted lines show the long and short axes of cells. $\mathrm{H}$ is the enlarged view of $\mathrm{G}$. (The boxed region in $\mathrm{H}$ shows stress fiber assay in H9c2 cells). I-J: Representative images of $0.1 \%$ DMSO and imidacloprid-treated H9c2 cells immunofluorescently-stained with Myh7, respectively. K: Bar chart showing the ratio of long axis to short axis. L: Bar chart shows cells containing stress fibers (\%). M: RT-PCR showing the expressions of CX43, p120, vinculin, Par3, ZO-1, a-actin, claudin1, claudin12 and RhoA at mRNA level in HH7 chick embryos exposed either $0.1 \%$ DMSO or imidacloprid. Scale bars $=200 \mu \mathrm{m}(\mathrm{B}, \mathrm{B} 1-\mathrm{B} 2, \mathrm{C}, \mathrm{C} 1-\mathrm{C} 2) ; 100 \mu \mathrm{m}(\mathrm{E}-\mathrm{J})$.

$$
215 \times 159 \mathrm{~mm} \text { ( } 300 \times 300 \text { DPI) }
$$


Imidacloprid

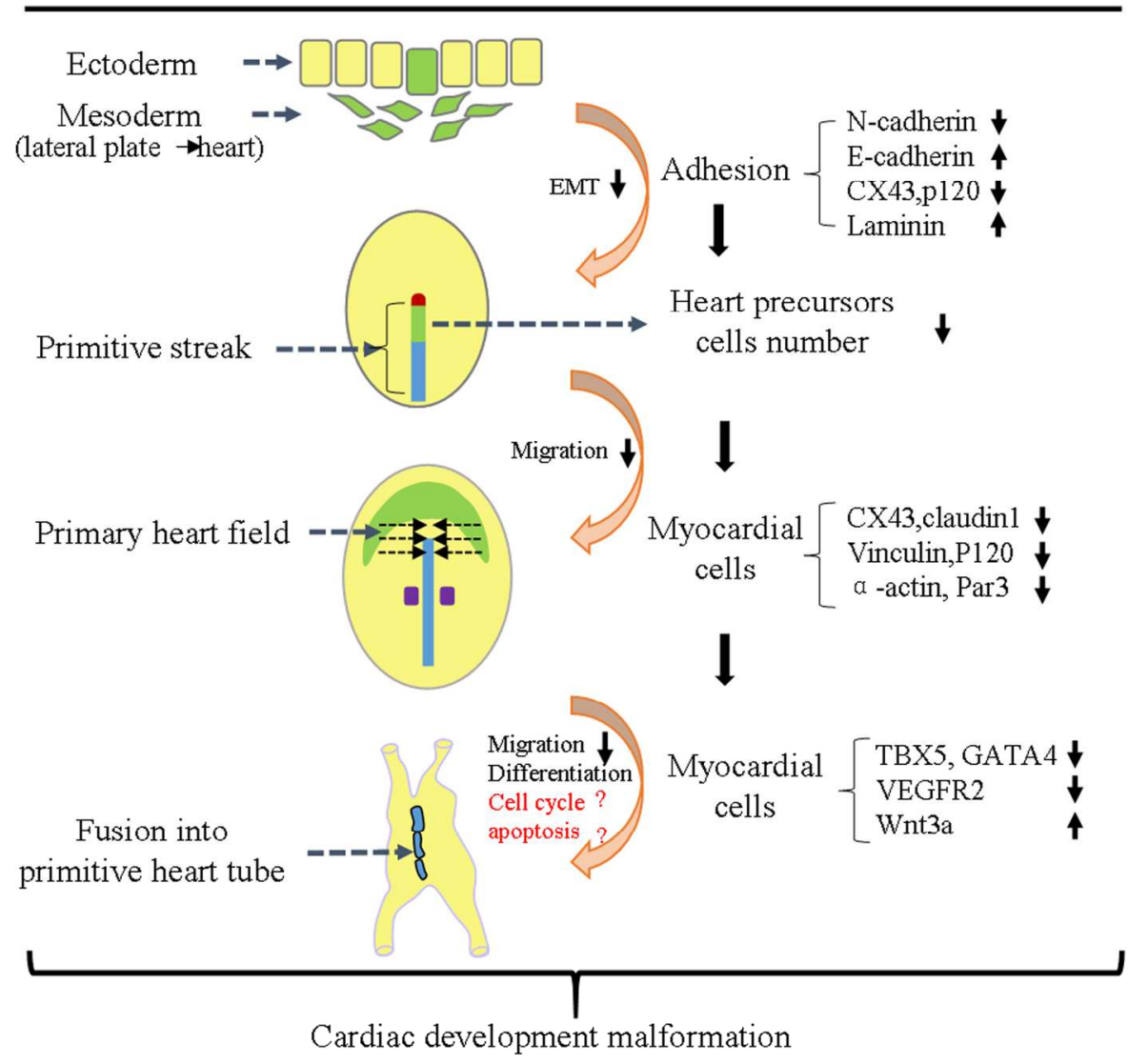

$181 \times 170 \mathrm{~mm}(300 \times 300 \mathrm{DPI})$ 


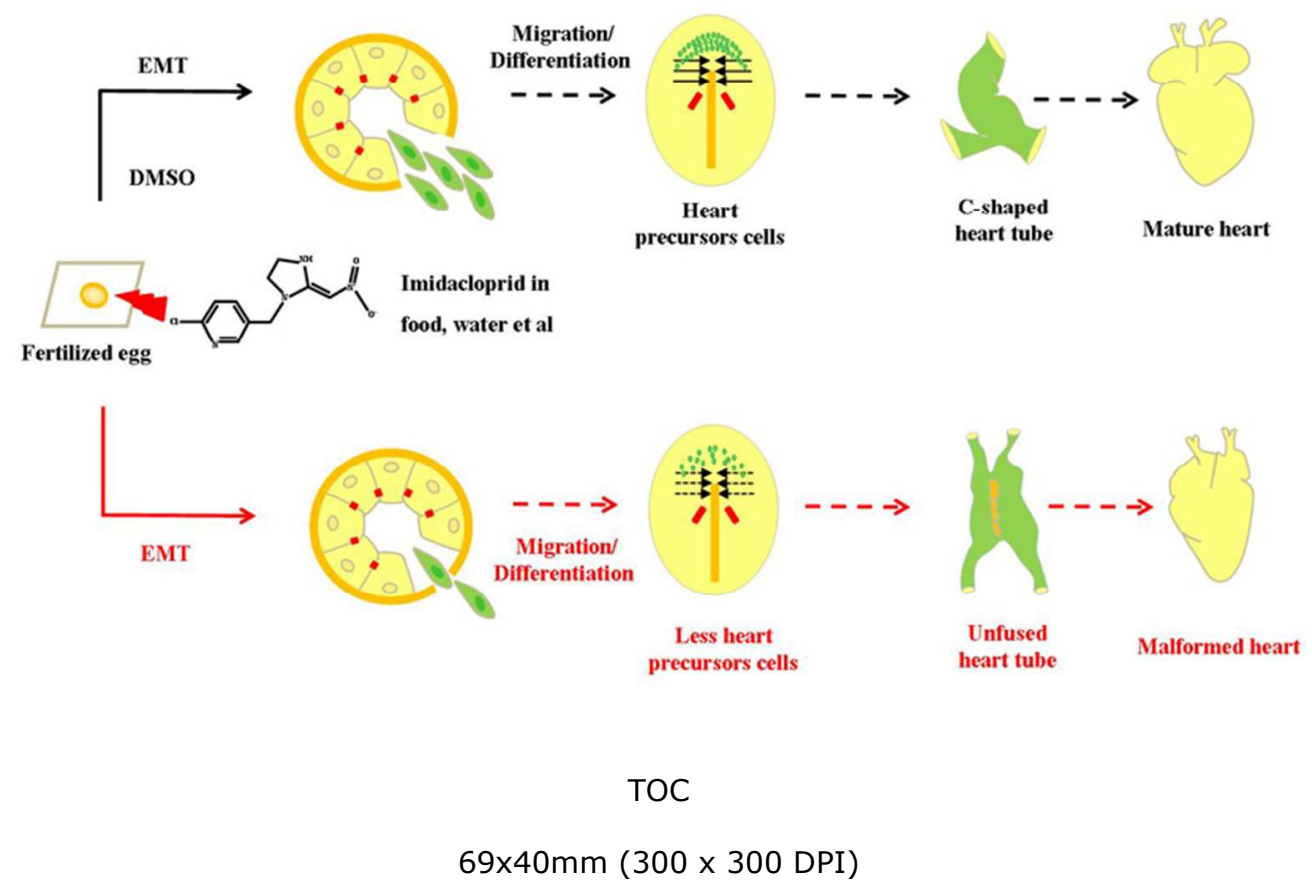

